Draft version August 19, 2021

Preprint typeset using $\mathrm{LAT}_{\mathrm{E}} \mathrm{X}$ style emulateapj v. 5/2/11

\title{
LAUNCHING AND QUENCHING OF BLACK HOLE RELATIVISTIC JETS AT LOW ACCRETION RATE
}

\author{
Hung-Yi Pu ${ }^{1}$, Kouichi Hirotani ${ }^{2}$, And Hsiang-Kunan Chang ${ }^{1,3}$ \\ Draft version August 19, 2021
}

\begin{abstract}
Relativistic jets are launched from black hole $(\mathrm{BH}) \mathrm{X}$-ray binaries and active galactic nuclei when the disk accretion rate is below a certain limit (i.e., when the ratio of the accretion rate to the Eddingtion accretion rate, $\dot{m}$, is below about 0.01 ) but quenched when above. We propose a new paradigm to explain this observed coupling between the jet and the accretion disk by investigating the extraction of the rotational energy of a $\mathrm{BH}$ when it is surrounded by different types of accretion disk. At low accretion rates (e.g., when $\dot{m} \lesssim 0.1$ ), the accretion near the event horizon is quasi-spherical. The accreting plasmas fall onto the event horizon in a wide range of latitudes, breaking down the force-free approximation near the horizon. To incorporate the plasma inertia effect, we consider the magnetohydrodynamical (MHD) extraction of the rotational energy from BHs by the accreting MHD fluid, as described by the MHD Penrose process. It is found that the energy extraction operates, and hence a relativistic jet is launched, preferentially when the accretion disk consists of an outer ShakuraSunyaev disk (SSD) and an inner advection-dominated accretion flow. When the entire accretion disk type changes into an SSD, the jet is quenched because the plasmas brings more rest-mass energy than what is extracted from the hole electromagnetically to stop the extraction. Several other observed BH disk-jet couplings, such as why the radio luminosity increases with increasing X-ray luminosity until the radio emission drops, are also explained.
\end{abstract}

Subject headings: accretion, accretion disks — black hole physics — Galaxies: active — magnetic fields - MHD - X-rays: binaries

\section{INTRODUCTION}

Black hole $(\mathrm{BH})$ relativistic jets are observed from black hole X-ray binaries (BHXBs) and active galactic nuclei(AGNs). A puzzling coupling between the jet and the accretion disk has been recognized. Observationally, the radio luminosity (which is likely due to the radiation from the relativistic jet or may also be from the semirelativistic disk wind) increases with increasing X-ray luminosity (which is likely due to the radiation from the disk), until it shows a sudden drop when the X-ray luminosity exceeds a certain limit (Gallo et al. 2003; Maccarone et al. 2003; Fender et al. 2004; Trump et al. 2011). In terms of the dimensionless accretion rate $\dot{m}$, this limit has a value about $\sim 0.01$, where $\dot{m} \equiv \dot{M} / \dot{M}_{\mathrm{Edd}}, \dot{M}$ is the accretion rate, $\dot{M}_{\mathrm{Edd}}=L_{\mathrm{Edd}} / \chi c^{2}$ is the Eddingtion accretion rate, $L_{\mathrm{Edd}}=1.38 \times 10^{38}\left(\mathrm{M} / M_{\odot}\right) \mathrm{erg} \mathrm{s}^{-1}$ is the Eddingtion luminosity, $\chi$ is the efficiency converting the energy of the accreting mass into the radiation energy, and $c$ is the speed of light. In addition, when the X-ray luminosity changes, the X-ray spectral state also changes, indicating that the accretion disk changes its type with the accretion rate (Fender et al. 2004; Trump et al. 2011; Esin et al. 1997; Remillard \& McClintock 2006). Since it is believed that BH relativistic jets are originated from the rotating $\mathrm{BHs}$ that are threaded by large-scale magnetic lines and are powered by the rotational energy of the BH (Blandford \& Znajek 1977), an investigation of whether the BH energy can be extracted by hole-threading magnetic field lines when the $\mathrm{BH}$ is surrounded by different type of accretion disks, may offers the key to an understanding of the disk-jet coupling of BHXBs and AGNs.

How the disk type varies $\dot{m}$ is described below. When the accretion rate is extremely low, the energy transfer due to Coulomb collisions between ions and electrons is very inefficient. As a result, the ions cannot efficiently transfer the heat to the electrons, and the disk becomes an advection-dominated accretion flow or ADAF for short (Naravan \& Yi 1995; Narayan et al. 1997; (Narayan et al. 1998), which corresponds to a hot, radiatively inefficient, optically thin and geometrically thick disk. As the accretion rate increases, the outer part of the disk cools down due to efficient Coulomb collisions, and becomes a cold, optically thick and geometrically thin disk (Shakura \& Sunyaev 1973; Novikov \& Thorne 1973), hereafter we use the term "SSD " (Shakura-Sunyaev disk) to refer to such disk; consequently, a combined disk is formed with an inner ADAF and an outer SSD. In a combined disk, the radius where the transition from thin disk to ADAF occurs is called the transition radius, $R_{\mathrm{tr}}$. In general, $R_{\mathrm{tr}}$ decreases with increasing $\dot{m}$ (Honma 1996; Manmoto et al. 2000). When the accretion rate further increases and exceeds a critical value with $\dot{m} \sim 0.01$, the Coulomb interaction becomes so efficient at every radius of the disk that the whole disk cools down, changing into the SSD. The plasma rotational motion is nearly Keplerian in a SSD, whereas it becomes sub-Keplerian in an ADAF, because the pressure gradient force contributed in the radial force balance in the latter type of accretion disk.

It is interesting to note that the accretion flow geometries near the horizon are quasi-spherical for all the above

\footnotetext{
${ }^{1}$ Department of Physics, National Tsing Hua University, Hsinchu 30013, Taiwan

2 Theoretical Institute for Advanced Research in Astrophysics, Academia Sinica, Institute of Astronomy and Astrophysics, P.O. Box 23-141, Taipei, Taiwan

${ }^{3}$ Institute of Astronomy, National Tsing Hua University, Hsinchu 30013, Taiwan
} 
disks (see Section 2 for more details). As a result, there are considerable plasma flow along the hole-threading line, reducing the electromagnetic extraction of $\mathrm{BH}$ energy by contributing their rest-mass energy. This consideration mainly differentiates our model from previous explanations of the disk-jet couplings (e.g., Meier 2005; Ferreira et al. 2006). We emphasize that this effect is important at low accretion rate, e.g., when $\dot{m} \lesssim 0.1$, due to the quasi-spherical geometry near the event horizon; at higher accretion rate, e.g., when $\dot{m}$ as large as $\geq 0.3$ (Abramowicz et al. 2010), the accretion disk type further varies to a slim disk (Abramowicz et al. 1988, 2010; Sadowski 2009) and a disk-type geometry near the horizon is realized. Therefore, a pure electromagnetic extraction of $\mathrm{BH}$ energy at most of the latitudes becomes a good approximation.

To consider the contribution of the quasi-spherical accreting plasma near the $\mathrm{BH}$, we adopt the magnetohydrodynamics (MHD) theory and consider the "MHD Penrose process" (Takahashi et al. 1990), which includes both the electromagnetic and plasma inertia effects, to examine the extraction of the $\mathrm{BH}$ rotational energy. By constraining the magnetic field strength and the plasma density from the theory of accretion disks in a strong gravitational field, we investigate the importance of plasma inertia for different accretion disk types at low accretion rates. It is found that the MHD extraction of BH energy takes place preferentially in a combined disk and therefore enables the launch of a relativistic jet; however, such relativistic jets are quenched if a magnetic dominance breaks down when the entire accretion disk become a thin disk. We also propose a new paradigm of the coupling between the accretion disk and the jet for both BHXBs and AGNs. The observed launching and quenching of relativistic jets at low accretion rates and several other puzzling observational results, are naturally explained.

We introduce our model in Section 2. In Section 3, we summarize necessary formulae and concepts of the general relativistic ideal MHD flow in a stationary and axisymmetric BH magnetosphere. Next, the result is given in Section 4. Finally, discussion and conclusion are respectively presented in Section 5 and 6.

\section{THE MODEL}

To qualitatively investigate whether a relativistic jet can be launched when the $\mathrm{BH}$ is surrounded by different type of accretion disks, we consider a model with following assumptions. Comments on the assumptions are provided in Section 5.3 .

1. Accretion disks are described by the analytical solutions adopted from the accretion disk theories. The accretion disk type is important since both the large-scale field strength and the mass flux per magnetic flux tube (hereafter, mass loading) are related to the disk. For simplicity, we adopt the analytical solutions of the ADAF and the SSD to represent the disk properties. For a combined disk, these two types of disk solutions are connected at the transition radius. The analytical solution we adopted is provided in the Appendix B. The 'middle region' solution of a SSD, in which the pressure and the opacity is respectively dominated by the gas pressure and the electron-scattering opacity, is used.

2. Parabolic large-scale hole-threading magnetic field lines are considered. We consider large-scale magnetic field lines that are dragged in from a distant region by the accretion flow. It is expected that the field lines can be finally accumulated near the $\mathrm{BH}$ with a parabolic geometry. Thus, instead of solving for the field geometry, we adopt the paraboloidal field solution given in Blandford \& Znajek (1977) for the magnetic flux function $A_{\phi}$,

$$
A_{\phi}=\frac{\mathcal{H}}{2}\{r(1-\cos \theta)+2(1+\cos \theta)[1-\ln (1+\cos \theta)]\}
$$

where the field strength $\mathcal{H}$ is to be determined (see Appendix $\mathrm{C}$ for more details). Here, we focus on magnetic field lines that thread the horizon, since they are responsible for the extraction of the rotational energy from a rotating BH.

3. The accretion flow has a quasi-spherical geometry near the event horizon. An accretion flow will have a quasispherical geometry near the horizon if the flow become transonic before the radius of the innermost stable circular orbit, $r_{\text {ISCO }}$, (Abramowicz \& Zurek 1981). Compare to a disk-like geometry, in which the accreting plasma enters the $\mathrm{BH}$ through a plunging region near the equatorial plane, the accreting plasma in a quasi-spherical geometry can fall onto the horizon at a wider range of latitudes. Since the sonic point of the accretion flow is well outside of $r_{\text {ISCO }}$ for a typical ADAF, which has $\alpha \gtrsim 0.2$ (Naravan et al. 1997), and the sonic point is close to $r_{\text {ISCO }}$ for an SSD, the plasma distribution near the horizon should have a quasi-spherical geometry for all the disk types we considered here, ADAF, combined disk, and SSD. Note that, for the SSD, the plasmas are conventionally supposed to plunge into the horizon in a disk-like geometry, which forms a striking contrast with our current picture.

4. The magnetic field strength is parameterized by the gravitational binding energy. Providing that the diffuse effect of the large-scale magnetic field is relatively unimportant, large-scale magnetic field lines can be 'arrested' by the accretion disk, being dragged from the outer region toward the central BH (Naravan et al. 2003; Spruit \& Uzdenskv 2005; Rothstein \& Lovelace 2008). It is convenient to parameterize its strength $B$ at radius $R$ by (see Appendix A)

$$
B \equiv B(\varepsilon, R, \sigma(R))=\varepsilon \sqrt{2 \pi G M_{\mathrm{BH}} \sigma / R^{2}},
$$

where $G$ is the gravitational constant, $M_{\mathrm{BH}}$ is the mass of the central $\mathrm{BH}, \sigma$ is the surface density of the disk, and $\varepsilon^{2}$ is the ratio of the gravitational binding energy of the disk at $R$ to the large-scale magnetic field energy inside radius $R$. The determination of $\varepsilon$ is not yet clear; however, it can be related to the ionization degree of the accretion flow and the magnetic Prandtl number.

4 In comparison, in the magnetically dominated limit, the Blandford-Znajek process (Blandford \& Znajek 1977) becomes a good approximation of the MHD Penrose process. 
5. The accretion disk type is a function of the accretion rate. As described in Section 1 , from low to high $\dot{m}$, the disk type varies as follows: (I) ADAF, (II) combined disk that consists of an inner ADAF and outer SSD, and (III) SSD. Hereafter, we call the corresponding range of $\dot{m}$ as "Range I", "Range II", and "Range III", respectively. Many studies on the transition radius $R_{\mathrm{tr}}$ between inner ADAF and outer SSD give similar results (Honma 1996; Manmoto et al. 2000). To qualitatively describe how the disk configuration changes with $\dot{m}$, we adopt the analytical solution of $R_{\mathrm{tr}}$ from Honma (1996)

$$
R_{\mathrm{tr}}=2.7648 \alpha^{4} \dot{m}^{-2} R_{\mathrm{g}} \quad \text { if } \dot{m} \leq \dot{m}_{\mathrm{cr}},
$$

where $\alpha$ is the viscosity of ADAF in the $\alpha$-prescription (Shakura \& Sunvaev 1973), $\dot{m}_{\mathrm{cr}}=\dot{M}_{\mathrm{cr}} / \dot{M}_{\mathrm{Edd}}$ is the critical accretion rate with a typical value $\leq 0.01$, beyond which there is no ADAF solution and hence the entire disk becomes the SSD type. We recognize that the entire disk becomes thin disk if $R_{\mathrm{tr}}<10 R_{\mathrm{g}}$ is met (Honma 1996; Manmoto et al. 2000), and define the critical accretion rate, $\dot{M}=\dot{M}_{\mathrm{cr}}$, such that $R_{\mathrm{tr}}=10 R_{\mathrm{g}}$ is obtained at $\dot{M}=\dot{M}_{\mathrm{cr}}$. By further assuming that the accretion disk has a finite outer radius $R_{\text {out }} \equiv 1000 R_{\mathrm{g}}$, different ranges can be defined by (see also Figure 1) Range I: $R_{\mathrm{tr}}>1000 R_{\mathrm{g}}$; Range II: $1000 R_{\mathrm{g}}>R_{\mathrm{tr}}>10 R_{\mathrm{g}}$; and Range III: $R_{\mathrm{tr}}<10 R_{\mathrm{g}}$.

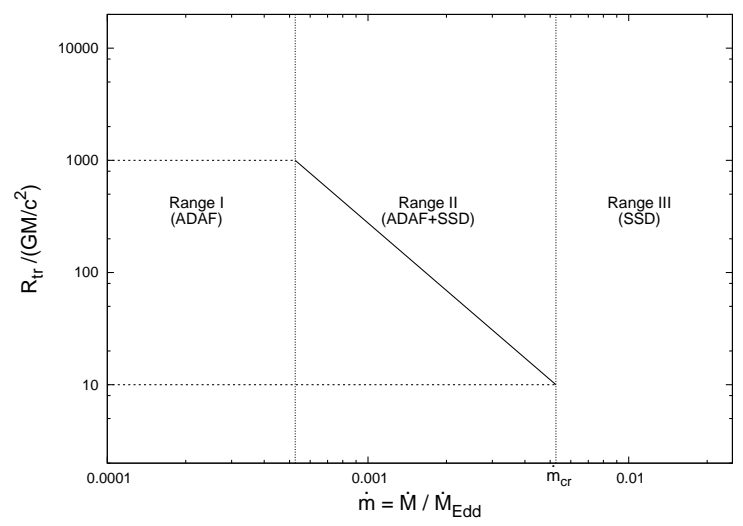

FIG. 1. - Disk type as a function of the accretion rate $\dot{m}$ in our model. The solid curve shows the computed value of Equation (3) with $\alpha=0.1$. In our calculation, we assume the accretion disk has a finite outer radius $R_{\mathrm{out}}=1000 R_{\mathrm{g}}$ and assume $R_{\mathrm{tr}}=10 R_{\mathrm{g}}$ when the accretion rate $\dot{m}$ equals to the critical accretion rate $\dot{m}_{\mathrm{cr}}$. Therefore, when $\dot{m}$ has a value within Range I/II/III, the disk type is of a(n) $\mathrm{ADAF} /$ combined disk/Shakura-Sunyaev disk (SSD), respectively. See the text for more details.

Our strategy to investigate the formation of relativistic jet at different disk types is described below. In contrast to that there is only outflow along the large-scale, disk-threading field lines for disk winds (Blandford \& Payne 1982; Sadowski \& Sikora 2010), there are both inflow and outflow along the hole-threading field lines. The inflow (or outflow) is launched when the gravitational force acting on the plasma is larger (or smaller) than the magnetocentrifugal forces do. Such a difference allows us to monitor the outflow properties by the inflow behavior; for an MHD flow along a holethreading line, a powerful outflow is realized only when the rotational energy is extracted by the inflow. Therefore, the condition for jet formation is to extract the rotational energy of $\mathrm{BH}$, which can be examined by solving the equation of motion, namely, that the relativistic Bernoulli equation (BE), of the inflow along the field line (see Section 3 for more details).

The influences of the disk type lie in how they affect the large-scale field strength $(\bar{B})$ and the mass loading. At the injection point of the inflow, $R_{i n j}$, by denoting the superscript "ADAF" and "SSD" as the analytical solutions of ADAF and SSD, respectively, and assuming that the same value of $\varepsilon$ can be applied to both ADAF and SSD, these two physical properties can be estimated (see Table 1) in different region of the computation domain of $\dot{m}$ accordingly.

Range I: ADAF type.- The large-scale field at $R_{\text {inj }}$ is estimated by the ADAF surface density, that is, $\bar{B}_{\mathrm{I}}=$ $B\left(\varepsilon, R_{\mathrm{inj}}, \sigma^{\mathrm{ADAF}}\right)$ by Equation (2). The mass flux per field line at $R_{\mathrm{inj}}$ is estimated by the ADAF solution as well, which gives $\rho^{\mathrm{ADAF}}\left(R_{\text {inj }}\right) u^{\mathrm{ADAF}}\left(R_{\text {inj }}\right) / \bar{B}_{\mathrm{I}}$.

Range II: Combined disk type. - The large-scale magnetic field at $R_{\text {inj }}$, which contains an additional term due to the magnetic field advected from the thin disk to the ADAF, is estimated by $\bar{B}_{\mathrm{II}}=B\left(\varepsilon, R_{\mathrm{inj}}, \sigma^{A \mathrm{DAF}}\right)+B\left(\varepsilon, R_{\mathrm{tr}}, \sigma^{\mathrm{SSD}}\right)$. The mass flux per magnetic flux tube at $R_{\text {inj }}$ is computed by $\rho^{\mathrm{ADAF}}\left(R_{\mathrm{inj}}\right) u^{\mathrm{ADAF}}\left(R_{\mathrm{inj}}\right) / \bar{B}_{\mathrm{II}}$, since the injection point is located inside the ADAF.

Range III: Thin disk type.- The large-scale field at $R_{\mathrm{inj}}$ is estimated by $\bar{B}_{\mathrm{III}}=B\left(\varepsilon, R_{\mathrm{inj}}, \sigma^{\mathrm{SSD}}\right)$ and the mass flux per field line at $R_{\text {inj }}$ is estimated by $\rho^{\mathrm{SSD}}\left(R_{\text {inj }}\right) u^{\mathrm{SSD}}\left(R_{\text {inj }}\right) / \bar{B}_{\text {III }}$.

In next section, we summarize necessary concepts of the ideal MHD flow in a BH magnetosphere, including the relativistic BE and the MHD Penrose process. The result of our model is provided in Section 4.

\section{MHD FLOWS AROUND A ROTATING BLACK HOLE}

Stationary and axisymmetric ideal MHD flows in a rotating BH magnetosphere have been investigated in several studies (Camenzind 1986a, b, 1987; Takahashi et al. 1990; Hirotani et al. 1992). In this paper, we adopt the Kerr 
TABLE 1

Estimating the Field Strength and the Mass Loading at Different Accretion Ranges

\begin{tabular}{cccc}
\hline \hline Range & Disk Type & Field Strength ${ }^{\mathrm{a}}$ at the Injection Point & Mass Loading at the Injection Point \\
\hline I & ADAF & $\bar{B}_{\mathrm{I}}=B_{\mathrm{inj}}\left(\varepsilon, R_{\mathrm{inj}}, \sigma^{\mathrm{ADAF}}\left(R_{\mathrm{inj}}\right)\right)$ & $\rho^{\mathrm{ADAF}} u^{\mathrm{ADAF}} / \bar{B}_{\mathrm{I}}$ \\
II & $\begin{array}{c}\text { ADAF+SSD } \\
(\text { Combined })\end{array}$ & $\begin{array}{c}\bar{B}_{\mathrm{II}}=B_{\mathrm{inj}}\left(\varepsilon, R_{\mathrm{inj}}, \sigma^{\mathrm{ADAF}}\left(R_{\mathrm{inj}}\right)\right) \\
+B_{t r}\left(\varepsilon, R_{\mathrm{tr}}, \sigma^{\mathrm{SSD}}\left(R_{\mathrm{tr}}\right)\right)\end{array}$ & $\rho^{\mathrm{ADAF}} u^{\mathrm{ADAF}} / \bar{B}_{\mathrm{II}}$ \\
III & $\mathrm{SSD}$ & $\bar{B}_{\mathrm{III}}=B_{\mathrm{inj}}\left(\varepsilon, R_{\mathrm{inj}}, \sigma^{\mathrm{SSD}}\left(R_{\mathrm{inj}}\right)\right)$ & $\rho^{\mathrm{SSD}} u^{\mathrm{SSD}} / \bar{B}_{\mathrm{III}}$
\end{tabular}

$a$ See Equation (2).

$b$ The mass flux per magnetic flux tube.

metric as the background geometry with signature $(-,+,+,+)$ and use the geometrized units such that $c=G=M=1$, that is, the length scale is in the unit of ' $G M / c^{2}$ '. Most of the derivations in this section follows the calculations in Takahashi et al. (1990). Note that a different signature $(+,-,-,-)$ is used in their paper.

\subsection{The Relativistic Bernoulli Equation}

In the Boyer-Lindquist coordinate, the metric is

$$
d s^{2}=-\frac{\Delta-a^{2} \sin ^{2} \theta}{\Sigma} d t^{2}-\frac{4 a r \sin ^{2} \theta}{\Sigma} d t d \phi+\frac{A \sin ^{2} \theta}{\Sigma} d \phi^{2}+\frac{\Sigma}{\Delta} d r^{2}+\Sigma d \theta^{2}
$$

where $a \equiv J, \Delta \equiv r^{2}-2 r+a^{2}, \Sigma \equiv r^{2}+a^{2} \cos ^{2} \theta$ and $A \equiv\left(r^{2}+a^{2}\right)^{2}+\triangle a^{2} \sin ^{2} \theta$. The ideal MHD condition requires that the electric field vanish in the fluid's rest frame,

$$
\sum_{\mu} F_{\mu \nu} u^{\mu}=F_{\mu \nu} u^{\mu}=0
$$

where $F_{\mu \nu}=A_{\nu, \mu}-A_{\mu, \nu}$ is the electromagnetic field tensor satisfying the Maxwell's equation, $u^{\nu}$ is the four velocity of the fluid, $A_{\mu}$ is the electromagnetic vector potential, and the comma refers to the derivative.

The fluid equation of motion $T_{; \nu}^{\mu \nu}=0$ can be split into the two components: the 'relativistic BE' (the poloidal equation) that describes the flow along the field line, and the 'Grad-Shafronov equation' (the trans-field equation) that describes the force balance perpendicular to the field lines, where the semi-colon refers to the covariant derivative. The BE can be obtained by projecting the equation of the motion along the field line; however, it can alternatively derived from the definition of proper time,

$$
u^{\alpha} u_{\alpha}=-1
$$

The energy-momentum tensor $T^{\mu \nu}=T_{\mathrm{em}}^{\mu \nu}+T_{\text {plasma }}^{\mu \nu}$ consists of two terms. One is the electromagnetic term,

$$
T_{\mathrm{em}}^{\mu \nu}=\frac{1}{4 \pi}\left(F^{\mu \alpha} F_{\alpha}^{\nu}-\frac{1}{4} g^{\mu \nu} F^{\alpha \beta} F_{\alpha \beta}\right)
$$

and the other is the plasma term,

$$
T_{\text {plasma }}^{\mu \nu}=(P+\rho) u^{\mu} u^{\nu}+g^{\mu \nu} P,
$$

where $P$ is the pressure and $\rho$ is the energy density. The proper number density $n$ obeys the continuity equation

$$
\left(n u^{\mu}\right)_{; \mu}=0
$$

and the relativistic specific enthalpy $\mu$ satisfies

$$
\mu=m_{\mathrm{p}}+\frac{\gamma}{\gamma-1} \frac{P}{n}=m_{\mathrm{p}}\left[1+\frac{\gamma}{\gamma-1} \frac{P_{\mathrm{inj}}}{n_{\mathrm{inj}} m_{\mathrm{p}}}\left(\frac{n}{n_{\mathrm{inj}}}\right)^{\gamma-1}\right]
$$

where $m_{\mathrm{p}}$ denotes the rest mass energy of the proton, $\gamma$ is the adiabatic index, and the subscript "inj" is the quantity evaluated at the particle injection point. The assumption of an adiabatic flow requires that the entropy along the field line be constant, which ensures that the term $P_{\text {inj }} /\left(n_{\text {inj }} m_{\mathrm{p}}\right)$ remains constant along the field line.

There are four more conserved quantities along the field line: the angular velocity of the field line, $\Omega_{\mathrm{F}}$, the particle flux per unit flux tube, $\eta$, the total energy of the flow per particle, $E$, and the total angular momentum of the flow per particle, L. They can defined by (cf. Equations(2.3)-(2.6) of Hirotani et al. 1992)

$$
\Omega_{\mathrm{F}}=\frac{F_{t r}}{F_{r \phi}}=\frac{F_{t \theta}}{F_{\theta \phi}}
$$




$$
\begin{gathered}
\eta=\frac{\sqrt{-g} n u^{r}}{F_{\theta \phi}}=\frac{\sqrt{-g} n u^{\theta}}{F_{\phi r}}=\frac{\sqrt{-g} n u^{t}\left(\Omega-\Omega_{\mathrm{F}}\right)}{F_{r \theta}}, \\
E=-\mu u_{t}-\frac{\Omega_{\mathrm{F}}}{4 \pi \eta} \sqrt{-g} F^{r \theta}=-\mu u_{t}+\frac{\Omega_{\mathrm{F}}}{4 \pi \eta} B_{\phi}, \\
L=\mu u_{\phi}-\frac{1}{4 \pi \eta} \sqrt{-g} F^{r \theta}=\mu u_{\phi}+\frac{B_{\phi}}{4 \pi \eta},
\end{gathered}
$$

where $-g \equiv-\operatorname{det}|g|=\Sigma^{2} \sin ^{2} \theta, \Omega \equiv u^{\phi} / u^{t}$ denotes the fluid angular velocity, and $B_{\phi} \equiv-\sqrt{-g} F^{r \theta}=$ $-(\Delta / \Sigma) \sin \theta F_{r \theta}$ the toroidal magnetic field seen by a distant static observer with four velocity $u_{\text {Lab }}^{\nu}=(1,0,0,0)$. One should note that the sign of $\eta$ is defined by the signs of $u^{r}$ and $F_{\theta \phi}$. Although the magnetic field is defined by

$$
B_{\mu} \equiv \frac{1}{2} \sqrt{-g} \epsilon_{\nu \mu \alpha \beta} F^{\alpha \beta} u_{\text {Lab }}^{\nu} ;
$$

however, it is convenient to introduce the rescaled poloidal field

$$
B_{\mathrm{P}}^{2} \equiv B^{A} B_{A}\left(g_{t t}+g_{t \phi} \Omega_{\mathrm{F}}\right)^{-2}=\frac{g^{A B}}{g_{t \phi}^{2}-g_{t t} g_{\phi \phi}}\left(A_{\phi, A} A_{\phi, B}\right),
$$

where $A$ runs over the poloidal coordinates, $r$ and $\theta$. Combining Equations (12)-(14), we have

$$
F^{r \theta} \frac{\sqrt{-g}}{4 \pi \eta}=-\frac{\left(g_{t \phi}+\Omega_{F} g_{\phi \phi}\right) E+\left(g_{t t}+\Omega_{F} g_{t \phi}\right) L}{M^{2}-K_{0}},
$$

where

$$
K_{0} \equiv-\left(g_{\phi \phi} \Omega_{\mathrm{F}}^{2}+2 g_{t \phi} \Omega_{\mathrm{F}}+g_{t t}\right),
$$

and the poloidal Alfven Mach number is

$$
M^{2}=4 \pi \mu n\left(\frac{u_{\mathrm{P}}}{B_{\mathrm{P}}}\right)^{2}=4 \pi \mu \eta\left(\frac{u_{\mathrm{P}}}{B_{\mathrm{P}}}\right)=4 \pi \mu \eta^{2} / n .
$$

The poloidal velocity of the plasma $u_{\mathrm{P}}$ is defined by

$$
u_{\mathrm{p}}^{2} \equiv u^{r} u_{r}+u^{\theta} u_{\theta}
$$

Substituting Equation (17) into Equations (13) and (14), we can express the energy of the fluid, $-\mu u_{t}$, and the angular momentum of the fluid, $\mu u_{\phi}$, as

$$
\begin{aligned}
& -\mu u_{t}=\frac{\left(g_{t t}+\Omega_{F} g_{t \phi}\right)\left(E-\Omega_{F} L\right)+M^{2} E}{M^{2}-K_{0}}, \\
& \mu u_{\phi}=-\frac{\left(g_{t \phi}+\Omega_{F} g_{\phi \phi}\right)\left(E-\Omega_{F} L\right)-M^{2} L}{M^{2}-K_{0}} .
\end{aligned}
$$

Finally, combining Equations (4), (20), (21), and (22), we obtain the BE (cf. Equation (17) of Takahashi et al. 1990),

$$
u_{\mathrm{p}}^{2}+1=\left(\frac{E}{\mu}\right)^{2} \frac{K_{0} K_{2}-2 K_{2} M^{2}-K_{4} M^{4}}{\left(M^{2}-K_{0}\right)^{2}}
$$

where $K_{2}$ and $K_{4}$ are defined as

$$
\begin{gathered}
K_{2} \equiv\left(1-\Omega_{\mathrm{F}} \frac{L}{E}\right)^{2} \\
K_{4} \equiv-\left[g_{\phi \phi}+2 g_{t \phi} \frac{L}{E}+g_{t t}\left(\frac{L}{E}\right)^{2}\right] /\left(g_{t \phi}^{2}-g_{t t} g_{\phi \phi}\right) .
\end{gathered}
$$

The BE, Equation (23), can be rewritten as a polynomial of $u_{P}$ with the aid of Equations (10) and (19). Note that the order of the polynomial equation depends on the second term of Equation (10), because particle number conservation gives $n \propto 1 / u_{\mathrm{p}}$. 


\subsection{The Cold Limit}

The cold limit can be adopted when the second term in Equation (10) is relatively unimportant to its first term, i.e., $P_{\text {inj }} / n_{\text {inj }} m_{\mathrm{p}} c^{2} \rightarrow 0$ (in c.g.s unit). Equation (10) therefore reduces to $\mu=m_{\mathrm{p}}$ and the BE becomes a fourth-order polynomial equation of $u_{\mathrm{P}}$. By introducing a parameter $\zeta \equiv u_{\mathrm{p}} / B_{\mathrm{p}}$, we have

$$
\begin{gathered}
\eta=n \zeta, \\
u_{\mathrm{p}}^{2}=\frac{\zeta^{2}}{\Delta \sin ^{2} \theta}\left[g^{r r}\left(A_{\phi, r}\right)^{2}+g^{\theta \theta}\left(A_{\phi, \theta}\right)^{2}\right], \\
B_{\mathrm{p}}^{2}=\frac{1}{\Delta \sin ^{2} \theta}\left[g^{r r}\left(A_{\phi, r}\right)^{2}+g^{\theta \theta}\left(A_{\phi, \theta}\right)^{2}\right], \\
M^{2}=4 \pi \mu n \zeta^{2}=4 \pi \mu \eta \zeta .
\end{gathered}
$$

The cold BE, therefore, can be expressed in terms of $\zeta$

$$
\left(\mathcal{A}^{2} \mathcal{B}\right) \zeta^{4}-\left(2 K_{0} \mathcal{A B}\right) \zeta^{3}+\left(\mathcal{A}^{2}-\mathcal{A}^{2} \mathcal{C}+K_{0}^{2} \mathcal{B}\right) \zeta^{2}+\left(2 \mathcal{D} \mathcal{A}-2 K_{0} \mathcal{A}\right) \zeta^{1}+K_{0}\left(K_{0}-\mathcal{D}\right)=0
$$

where

$$
\begin{gathered}
\mathcal{A}=4 \pi \mu \eta \\
\mathcal{B}=\frac{u_{P}^{2}}{\zeta^{2}}=B_{p}^{2}, \\
\mathcal{C}=-\left(\frac{E}{\mu}\right)^{2} K_{4}, \\
\mathcal{D}=\left(\frac{E}{\mu}\right)^{2} K_{2} .
\end{gathered}
$$

Even for a disk whose temperature is comparable with the virial temperature and the internal energy is comparable to the released gravitational energy (e.g., ADAF), the cold limit can still be modestly satisfied. This is because the released gravitational binding energy is still a small fraction of the rest-mass energy. Therefore, we solve the BE in cold limit, Equation (26), for simplicity and expect the result is at least qualitatively correct. In order to specify the coefficients $\mathcal{A}$ and $\mathcal{B}$, we need to specify the mass loading (mass flux per magnetic flux tube), $\mu \eta$, and the strength and geometry of the magnetic field. It is provided in the Appendix $\mathrm{C}$ how we compute these coefficients.

\subsection{Light Surfaces and the Separation Point}

There are two light surfaces, which are defined by $K_{0}=0$, in a BH magnetosphere. The outer light surface is formed by the centrifugal force in the same manner as the light cylinder in a pulsar magnetosphere, and the inner light surface is, on the other hand, formed by the strong gravity of the $\mathrm{BH}$ and the rotation of the magnetic field.

If a plasma starts with a negligible poloidal velocity, the inflow and outflow along the field line separate at the point $r_{\mathrm{s}}$ where the gravitational force balances with the magnetocentrifugal forces. This point is called the "separation point" (or the "stagnation point") and defined by $K_{0}^{\prime}=0$, which leads to $\left(\ln u_{P}\right)^{\prime}=0$, where the prime denotes the derivative along the flow line. That is, if a plasma is injected at $r=r_{\text {inj }}$ with $r_{\text {inj }}<r_{\mathrm{s}}$ (or with $r_{\text {inj }}>r_{\mathrm{s}}$ ), it will be accelerated inward (or outward), subsequently passing through the Alfven point $r=r_{\mathrm{A}}$ and the inner (or outer) light surface. Figure 3 shows the contours of $K_{0}$ around a $\mathrm{BH}$ with spin parameter $a=0.6$. Note that, at the injection point, the BE reduces to

$$
E-\Omega_{\mathrm{F}} L=\left.\mu \sqrt{K_{0}}\right|_{r_{\mathrm{inj}}}
$$

\subsection{Critical Points}

The critical points for an MHD flow can be found by differentiating Equation $(23)$ along the flow line, $\left(\ln u_{P}\right)^{\prime}=N / D$, where explicit expressions of $N$ and $D$ are given in Camenzind (1986b) and Takahashi et al. (1990). For a general MHD inflow, there are three critical points at which both $D$ and $N$ vanish: the 'slow-magnetosonic point', the 'Alfven point', and the 'fast-magnetosonic point'; the poloidal velocity $u_{\mathrm{p}}$ there matches the slow-magnetosonic velocity, the Alfven velocity, and the fast-magnetosonic velocity, respectively. In the cold limit, the slow-magnetosonic velocity reduces to zero. Due to the causality requirement at the event horizon, any MHD inflow must pass through the fast-magnetosonic point, after passing through the Alfven point.

At the Alfven point $r=r_{\mathrm{A}}$, the Mach number equals $K_{0}$, 


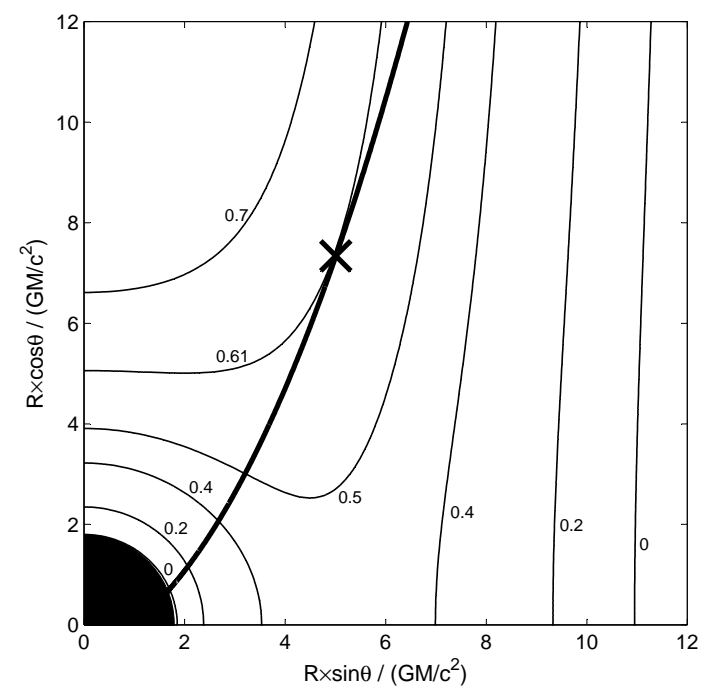

FIG. 2. - Illustration of the separation point of a field line and contours of $K_{0}$ around a black hole with spin parameter $a=0.6$ and $\Omega_{F}=0.5 \Omega_{H}$. Light surfaces are defined by $K_{0}=0$. The Alfven point of a physical MHD flow must be located inside the two (inner and outer) light surfaces, because $\left.M^{2}\right|_{r_{A}}=\left.K_{0}\right|_{r_{A}} \geq 0$. Black region represents the BH. For a field line threading the BH (black solid line), the separation point is determined by $K_{0}^{\prime}=0$ (indicated by a cross). See the text for more details.

$$
\left.M^{2}\right|_{r_{\mathrm{A}}}=\left.K_{0}\right|_{r_{\mathrm{A}}} .
$$

Note that the requirement of $\left.M^{2}\right|_{r_{\mathrm{A}}}=\left.K_{0}\right|_{r_{\mathrm{A}}} \geq 0$ implies that $r_{\mathrm{A}}$ must be located between these two light surfaces. Besides, it is convenient to express $E$ and $L$ in terms of the quantities evaluated at the Alfven point. Since both $D$ and $N$ automatically vanish at the Alfven point, no additional constraints are imposed on the MHD flow at the Alfven point. Therefore, by requiring that the numerators in Equations (21)-(23) vanish at $r=r_{\mathrm{A}}$, we obtain

$$
\begin{gathered}
E=-\left.\frac{\left(g_{t t}+\Omega_{\mathrm{F}} g_{t \phi}\right)\left(E-\Omega_{F} L\right)}{M^{2}}\right|_{r_{\mathrm{A}}}, \\
L=\left.\frac{\left(g_{t \phi}+\Omega_{\mathrm{F}} g_{\phi \phi}\right)\left(E-\Omega_{F} L\right)}{M^{2}}\right|_{r_{\mathrm{A}}},
\end{gathered}
$$

and

$$
\left.K_{2}\right|_{r_{\mathrm{A}}}=-\left.K_{2} K_{0}\right|_{r_{\mathrm{A}}} .
$$

Combining Equations (27), (29), and (30), we can express the conserved quantities $E$ and $L$ in terms of $r_{\mathrm{inj}}$ and $r_{\mathrm{A}}$.

After the MHD inflow pass through the Alfven point, $D$ vanishes again in $\left(\ln u_{P}\right)^{\prime}=N / D$ at the fast-magnetosonic point; thus, $N$ should also vanish there. We should notice here that both $D=0$ and $N=0$ can be satisfied at the fast-magnetosonic point only for a specific combination of the conserved quantities, $\Omega_{\mathrm{F}}, E$, $L$, and $\eta$. In previous works, e.g., Camenzind (1986a) and Takahashi et al. (1990), the authors gave $\Omega_{\mathrm{F}}, E$, and $L$ (or equivalently, $\Omega_{\mathrm{F}}, r_{\mathrm{inj}}$, and $r_{\mathrm{A}}$ ) and search $\eta$ to select the trajectory that pass through the fast-magnetosonic point. In this work, we instead give $\Omega_{\mathrm{F}}, r_{\mathrm{inj}}$, and $\eta$, considering plasma injection from the innermost region of the accretion disk, and solve for $r_{\mathrm{A}}$ (or equivalently, $E$ ). As explained next, whether the rotational energy of the $\mathrm{BH}$ is extracted outward can be determined by the location of $r_{\mathrm{A}}$.

\subsection{Negative Energy Flow}

The sign of $E$ can be solely determined by $r_{\mathrm{A}}$ via Equation (29) because it follows form Equation (27) that $E-\Omega_{\mathrm{F}} L>$ 0 holds for any MHD flow starting from a position between the two light surfaces with a vanishing poloidal velocity. Note that $E$ becomes negative if $\left.\left(g_{t t}+\Omega_{\mathrm{F}} g_{t \phi}\right)\right|_{r_{A}}>0$. Thus, near the BH, a spatial region called the "negative energy region", which satisfies $\left(g_{t t}+\Omega_{\mathrm{F}} g_{t \phi}\right)>0$, can be defined (Takahashi et al. 1990). If the Alfven point resides in this region, the MHD inflow has $E<0$ (which also implies $L<0$ because of $E-\Omega_{\mathrm{F}} L>0$; whereas $L<0$ does not imply $E>0)$.

A spinning BH differs from a static BH by an additional structure called "ergosphere" (which is defined by $g_{t t}>0$ ), a region inside which any particle must orbit in the same direction of the hole spin. Being locating outside the horizon, the ergosphere can be viewed as the region that stores the rotation energy of a BH. Note that the negative energy region exists only when $\Omega_{H}>\Omega_{F}>0$, and it is always located inside the ergosphere (see also Figure 3). It is also noteworthy that a negative energy MHD inflow can be launched from a region outside the ergosphere. 


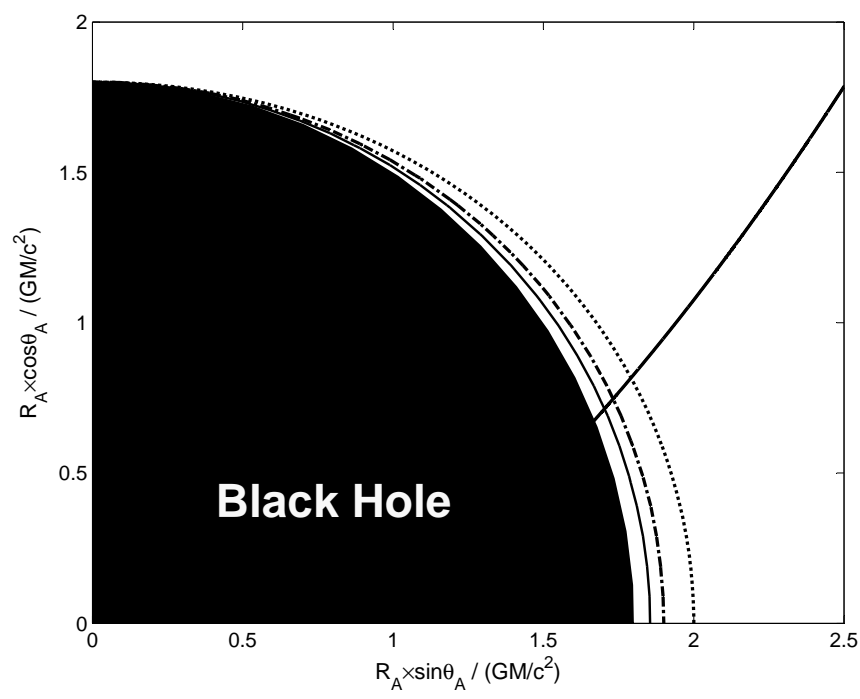

FIG. 3. - Spatial position of the Alfven point that gives a negative energy flow for the case of $\Omega_{F}=0.5 \Omega_{H}$ and $a=0.6$. The dash-dot line, which is defined by $g_{t t}+\Omega_{F} g_{t \phi}=0$, is located between the inner light surface (thin solid line) and the static limit (dotted line), the latter which defines the outer boundary of the ergosphere. An MHD flow has a negative energy if the Alfven point is located within the negative-energy region, which is defined as the region between the dash-dot and the thin solid lines. The magnetic field threading the hole surface in Figure 2 is also shown (thick sold line). See also Figure 6 of Takahashi et al. (1990).

TABLE 2

Inflow Energy as the Criterion For ERgospheric Jet LAUnch

\begin{tabular}{|c|c|c|c|}
\hline Inflow Property & Infow Energy ${ }^{b}$ & Inflow Energy Flux ${ }^{\mathrm{c}}$ & Jet Launch? ${ }^{\mathrm{d}}$ \\
\hline plasma dominated $\left(\mathcal{E}_{\mathrm{em}}^{r}<\left|\mathcal{E}_{\text {plasma }}^{r}\right|\right)^{\mathrm{a}}$ & $E>0$ & $\mathcal{E}^{r}<0$ & $\mathrm{NO}$ \\
\hline magnetically dominated $\left(\mathcal{E}_{\mathrm{em}}^{r}>\left|\mathcal{E}_{\text {plasma }}^{r}\right|\right)$ & $E<0$ & $\mathcal{E}^{r}>0$ & YES \\
\hline
\end{tabular}

\subsection{Outward Energy Flux and the MHD Penrose Process}

The outward energy flux of the flow $\mathcal{E}^{r}$ can be split into the electromagnetic part $\mathcal{E}_{\text {em }}^{r}$ and the fluid part $\mathcal{E}_{\text {plasma }}^{r}$,

$$
\mathcal{E}^{r} \equiv-T_{t}^{r}=n E u^{r}=\mathcal{E}_{\text {em }}^{r}+\mathcal{E}_{\text {plasma }}^{r}
$$

where,

$$
\begin{gathered}
\mathcal{E}_{\mathrm{em}}^{r} \equiv-\frac{1}{4 \pi} F^{r \theta} F_{t \theta}=-\frac{\Omega_{F}}{4 \pi} F^{r \theta} A_{\phi, \theta}=\frac{\Omega_{F}}{4 \pi} \frac{B_{\phi}}{\Sigma \sin \theta} A_{\phi, \theta} . \\
\mathcal{E}_{\text {plasma }}^{r} \equiv-n \mu u_{t} u^{r} .
\end{gathered}
$$

Therefore, a negative energy inflow $\left(E<0\right.$ and $\left.u^{r}<0\right)$ results in a positive outward energy flux $\left(\mathcal{E}^{r}>0\right)$, that is, the $\mathrm{BH}$ rotational energy is extracted. Note that $\mathcal{E}_{\text {om }}^{r}>0$ usually holds because its sufficient condition, $0<\Omega_{F}<\Omega_{H}$, is satisfied under normal conditions (MacDonald \& Thorne 1982), where $\Omega_{H}$ refer to the rotational angular velocities of the BH. Note also that the plasmas usually cannot extract BH's energy (i.e., $\mathcal{E}_{\text {plasma }}^{r}<0$ usually holds) because of their rest-mass contribution, unless they are in a negative energy orbit, which is rare.

In a magnetically dominated magnetosphere $\left(\mathcal{E}_{\mathrm{em}}^{r}>\mathcal{E}_{\text {plasma }}^{r}\right)$, because $\mathcal{E}^{r}$ is continuous across the separation point, a stationary outflow solution $\left(u^{r}>0, E>0\right.$ and $\left.\mathcal{E}^{r}>0\right)$ is possible only when it connects a negative-energy inflow ( $E<0$ and $u^{r}<0$, such that $\mathcal{E}^{r}>0$ ). Consequently, a stationary MHD jet from a BH ergosphere ( a "ergospheric jet") can be launched in a magnetically dominated magnetosphere only when the MHD Penrose process, $\mathcal{E}^{r}>0$, operates. If a magnetic dominance breaks down, $\mathcal{E}^{r}$ can be discontinuous across the separation point, that is, an outflow $\left(\mathcal{E}^{r}>0\right)$ can connect to a positive-energy inflow $\left(\mathcal{E}^{r}<0\right)$, owing to the energy supplied by the accreting plasma near the separation point. However, this kind of outflow, similar to the disk wind, will not be accelerated into relativistic energies and therefore is not of interest in the present paper. To summarize, for an MHD flow along the hole-threading field line, a stationary outflow with positive outward energy flux $\left(\mathcal{E}^{r}>0\right)$ should connect an inflow 
$\mathrm{BHXB}$

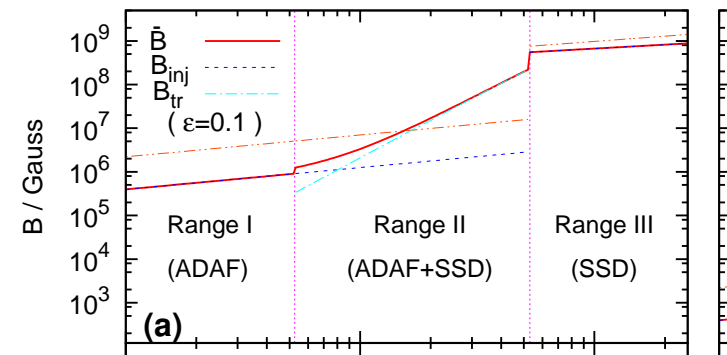

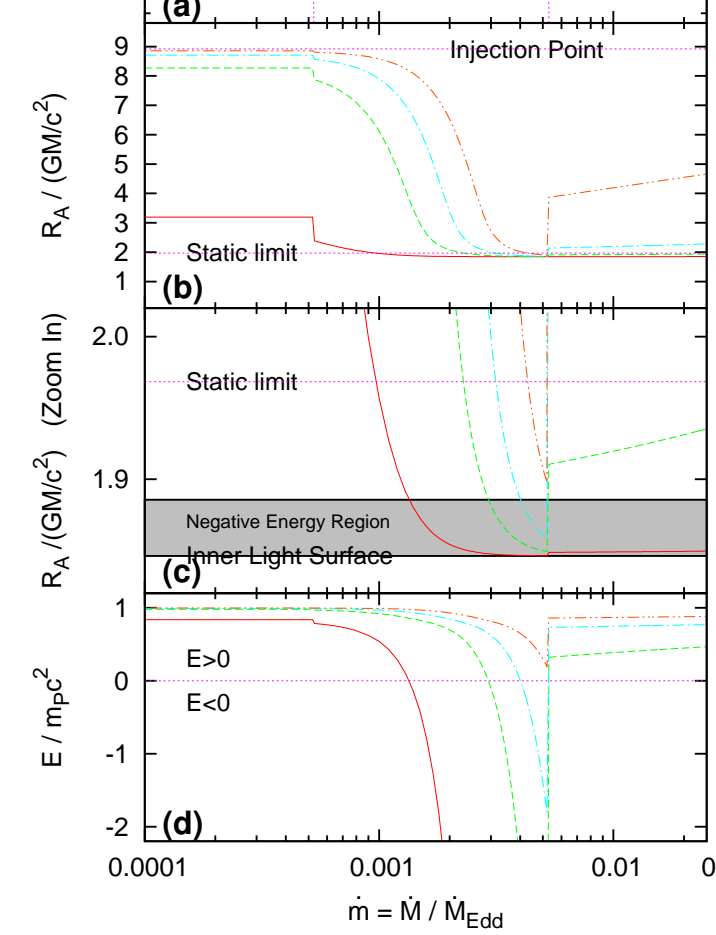

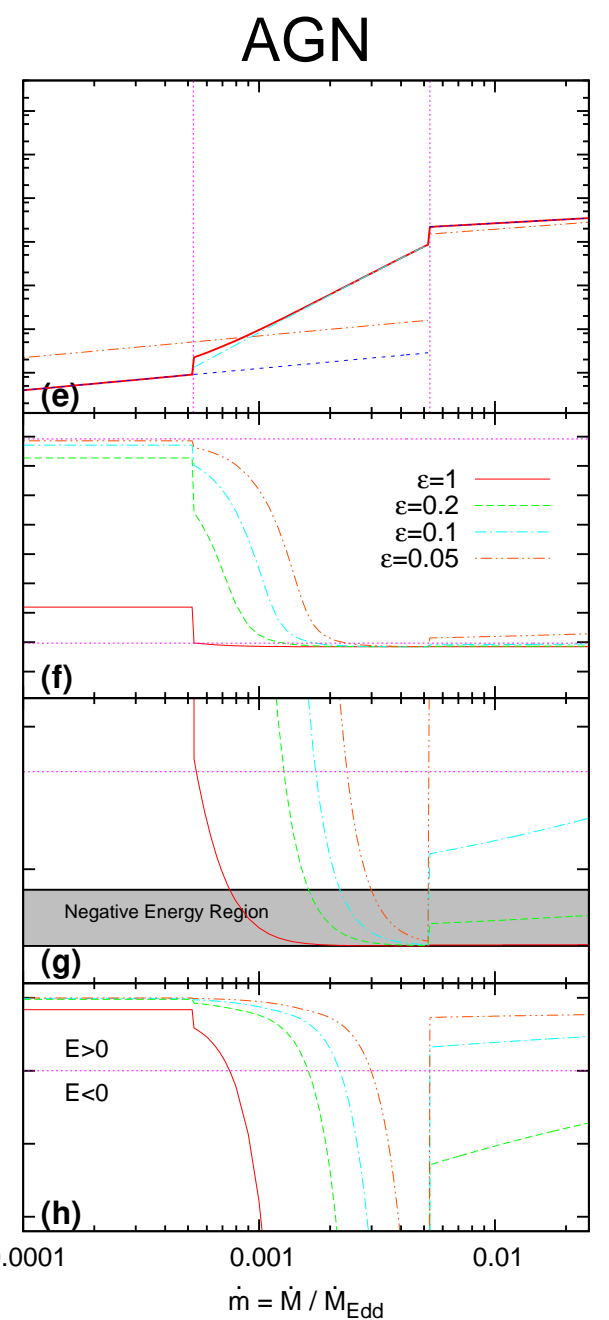

FIG. 4.- Extraction of BH rotational energy by MHD inflow (left column: for a BH mass of $10 M_{\odot} ;$ right column: $10^{6} M_{\odot}$ ). The domain of accretion rate $\dot{m}$ is divided into three Ranges (I, II and III) according to the disk types. The parameter $\varepsilon(\leq 1)$ is the squared root of the ratio between the large-scale magnetic field energy and the disk gravitational binding energy; that is, $\varepsilon$ denotes the ability for a disk to confine the large-scale field. (a) and (e) Large-scale magnetic field strength $B$ (solid line) at the injection point. In Ranges I and III, only $B_{\text {inj }}$ (dashed line) contributes to $\bar{B}$, where $B_{\text {inj }}$ denotes the field strength evaluated at the injection point. In Range II, an additional term $B_{\mathrm{tr}}$ (dash-dotted line) is added so that the fields advecting from the outer SSD into the inner ADAF region may be taken into account, where $B_{\operatorname{tr}}$ denotes the field strength evaluated at the transition radius of the SSD and the ADAF (see Section 2 for more details). For comparison, we also plot the strength of local magnetic field when the plasma beta becomes 1 at the injection point as the dash-dot-dot line. To avoid complexity, only the case of $\varepsilon=0.1$ is shown here, while $\varepsilon=1,0.2,0.1$ and 0.05 are shown in the rest of panels. (b) and (f) The location of the Alfven point $R_{\mathrm{A}}$. (c) and (g) Zoom-in plot of the second panel, (b) and (f), near the ergosphere. The static limit indicates the outer boundary of the ergosphere. The flow energy becomes negative if $R_{\mathrm{A}}$ is located inside the 'negative energy region' indicated by the shaded region (see also Figure 3). (d) and (h) Total energy (consisting of plasma and magnetic energies) per particle in unit of particle rest mass energy, $m_{\mathrm{p}} c^{2}$. Unless $E \approx m_{p} c^{2}$, the magnetosphere becomes magnetically dominated.

solution with $\mathcal{E}^{r}>0$ at the separation point, provided that the injected energy flux by accretion is small compared to the Poynting flux. Thus, we can investigate the activity of a Poynting-flux-dominated jet (or an outflow) by examining the inflow energy, $E$ (see also Table 2).

It should be noted here that the term "MHD Penrose process" refers to the case of $\mathcal{E}^{r}>0$ (remember $E$ is the 'total' energy, consisting both the electromagnetic plus plasma energy of the flow), as initially proposed by Takahashi et al. (1990); however, it was used to indicate $\mathcal{E}_{\text {plasma }}^{r}>0$ (i.e., a negative energy orbit of the particle) in some previous studies (Hirotani et al. 1992; Koide et al. 2002; Semenov et al. 2004; Komissarov 2005). The latter case is possible, but can only be transient (Koide et al.|2002; Komissarov 2005). In comparison, in the original idea of the 'Penrose process' (Penrose 1969), only the particle contribution $\left(\mathcal{E}^{r}=\mathcal{E}_{\text {plasma }}^{r}>0\right.$ ) was considered; whereas in the Blandford-Znajek process (Blandford \& Znajek 1977), only the electromagnetic contribution $\left(\mathcal{E}^{r}=\mathcal{E}_{\mathrm{em}}^{r}>0\right)$ was considered.

\section{RESULT}


We choose representative parameters to demonstrate the jet behavior at different disk type qualitatively. Because the extraction of the rotational energy of $\mathrm{BH}$ is mainly related to whether the $\mathrm{BH}$ magnetosphere is magnetically dominated or not, the change of the parameters will result in only minor modification of the result in our model.

We adopt the dimensionless BH spin parameter to be 0.6 and the angular velocity of the field, $\Omega_{\mathrm{F}}$, to be half of the angular velocity of the hole, $\Omega_{\mathrm{H}}$ (i.e., $\Omega_{\mathrm{F}}=0.5 \Omega_{\mathrm{H}}=0.0833$ ). The MHD inflow along the field line that intersects the event horizon at a modest latitude $\left(\theta_{\mathrm{H}} \sim 1.1868 \mathrm{rad}\right.$, i.e., about $68^{\circ}$ from the pole; see Figures 2 and 3 for this field line) is considered, and the inflow is assumed to be launched at the separation point (that is, the injection point is located at the separation point). In addition, to investigate the importance of the $\mathrm{BH}$ mass, we consider separately the BHXB and AGN cases. For BHXB, we assume $M_{\mathrm{BH}}=10 M_{\odot}$, whereas for AGN, $M_{\mathrm{BH}}=10^{6} M_{\odot}$. Furthermore, to explore the dependence of the ability of the disk in storing the large-scale magnetic field, we adopt different values of $\varepsilon(\varepsilon=1,0.2,0.1$, and 0.05$)$.

After starting from the injection point, the accreting MHD plasma is accelerated inward, passing thorough the Alfven point, $R_{\mathrm{A}}$, and the fast-magnetosonic point, and finally enter the $\mathrm{BH}$. The inflow energy $E$ can be computed by imposing the fast-magnetosonic condition once the injection point, the angular velocity of the field and the mass loading are specified. However, to discuss the conditions for jet launching $\left(\mathcal{E}^{r}>0\right)$ and quenching $\left(\mathcal{E}^{r}<0\right)$, it is adequate to examine the sign of $E$, which is uniquely specified by the location of $R_{\mathrm{A}}$. Specifically, if $R_{\mathrm{A}}$ resides in the negative energy region, the MHD inflow has a negative energy $(E<0)$ and the MHD Penrose process operates. Therefore, by solving $R_{\mathrm{A}}$ from the $\mathrm{BE}$ for an MHD inflow onto a $\mathrm{BH}$, we can investigate whether the energy extraction takes place as a function of $\dot{m}$. Detailed computation steps are provided in the Appendix.

Let us first consider the BHXB case with the BH mass, $M_{\mathrm{BH}}=10 M_{\odot}$ (Figures 4(a)-(d)). Figure 4(a) shows how the large-scale field strength at the injection point varies with the accretion rate. It is important to note that in a combined disk (Region II), the surface density of the disk does not monotonically increase inward but has a local maximum at the transition radius, $R_{t r}$. Since an SSD can confine more large-scale field than an ADAF because of its greater surface density for a fixed $\varepsilon$, the large-scale field arrested in the inner ADAF for a combined disk (solid line) is relatively stronger than what the ADAF alone would bring into the $\mathrm{BH}$ (dashed line). The locations of $R_{\mathrm{A}}$ for four discrete values of $\varepsilon$ are shown in Figures 4(b) and 4(c); the solid, dashed, dash-dotted, and dash-dot-dot lines correspond to $\varepsilon=1,0.2,0.1$, and 0.05 , respectively. It follows from the zoom-in figure (Figure $4(\mathrm{c})$ ) that $R_{\mathrm{A}}$ enters the negative energy region (indicated by the shaded region) in Range II for $\varepsilon=1,0.2$, and 0.1 , indicating that the MHD Penrose process turns on in a combined disk. This is because, near the horizon, the relatively strong magnetic field (which is mainly provided by the outer SSD) and the relatively small mass loading (which is provided by the inner ADAF) result in a magnetically dominated environment. In comparison, a magnetically dominated environment near the horizon is relatively harder to achieved when the disk is an ADAF (Range I) or an SSD (Range III), because it is the same type of disk responsible for both the confinement of the large-scale field and the loading of the plasma. Such disk-jet coupling provides the reason why observed relativistic jet is launched when $\dot{m}$ is near (but smaller) the value 0.01 , but quenched when $\dot{m}$ exceeds $\sim 0.01$. In addition, since $\bar{B}$ increases with increasing $\dot{m}, E$ increases negatively (i.e., the rotational energy of $\mathrm{BH}$ is more efficiently extracted outwards) with increasing $\dot{m}$ once $R_{A}$ enters the negative energy region in Range II (Figure 4(d)). This is because the BH magnetosphere becomes more magnetically dominated when the transition radius decreases with $\dot{m}$, resulting from that the difference between the surface density of the outer SSD and inner ADAF becomes more significant.

Next, let us consider the AGN case with $M_{\mathrm{BH}}=10^{6} M_{\odot}$ (Figures 4(e)-(h)). Although the solutions look qualitatively similar, there are three major differences from the BHXB case. First, comparing Figures 4(b), (c), (f) and (g), we find that for an AGN, $E<0$ is realized in a wider range of $\dot{m}$ for a fixed $\varepsilon$ or in a wider range of $\varepsilon$ for a fixed $\dot{m}$. Second, comparing Figures 4(d) and (h), we find that an AGN can extract more BH rotational energy per plasma particle than a BHXB can. Third, in an AGN, the MHD Penrose process works also in an SSD (i.e., Range III) for a relatively greater $\varepsilon$ (e.g., $\varepsilon>0.2$ ). However, the jet power decreases with increasing $\dot{m}$ in an SSD (i.e., Range III), in contrast to the case of a combined disk (i.e., Range II). The above comparison can help to understand the observed properties of accreting $\mathrm{BH}$ in different masses (see below).

\section{DISCUSSION}

\subsection{Universal Paradigm of Black Hole Disk-Jet Coupling at Low Accretion Rate}

The results in Section 4 can offer the key to an understanding of the observed connections between the disks and the jets for BHXBs and AGNs. A universal paradigm of disk-jet couplings on the $\dot{m}-E$ plane (c.f. Figures $4 \mathrm{~d} \&$ $4 \mathrm{~h}$ ) is diagrammatically presented in Figure 5. Transitions of disk-jet states are indicated by the arrows for three representative values of $\varepsilon$. Since the terminal speed of an MHD outflow is roughly determined by the magnetic dominance at the launching point, the more magnetically dominated the disk innermost region becomes (i.e., the more a system moves downward on the $\dot{m}-E$ plane), the greater speed an MHD outflow achieves. This discussion is valid for both the disk wind near the BH and the ergospheric jet. The two downward arrows in the right edge of this figure indicate how the terminal speed changes for a disk wind and a relativistic jet. Note that the ergospheric jet can only be launched when $E<0$ (i.e., in the lower half of the diagram).

The transitions of disk-jet states for BHXBs, which are indicated by the solid arrows, explain the observed features of the BHXB disk-jet couplings. Fender et al. (2004) show that two type of outflows associated with the X-ray spectral types are required to explain the systematic properties of the radio emission of BHXBs. The first type is a steady outflow observed when the BHXB is in a hard state (HS), while the second type is a transient, ballistic, powerful 


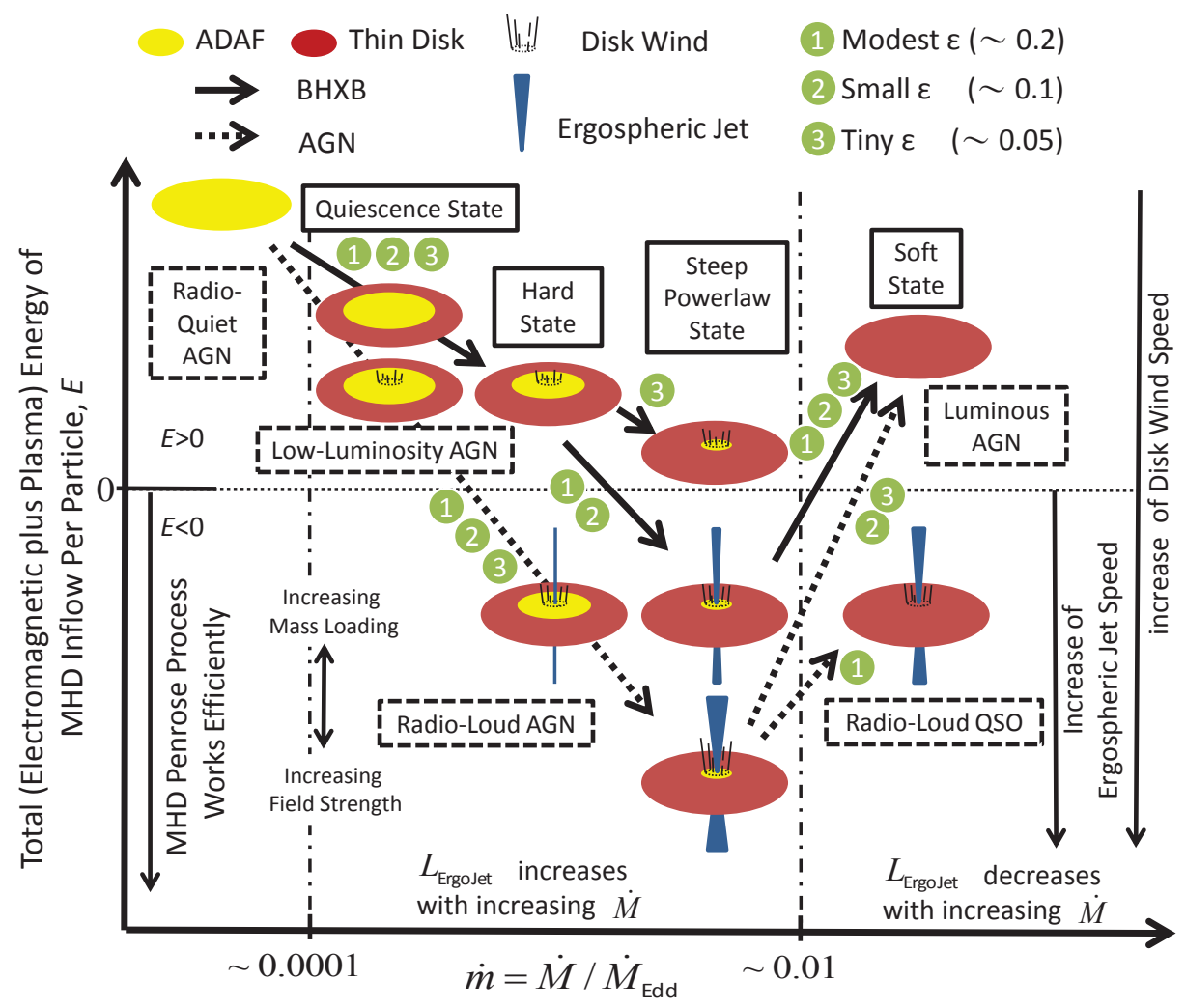

FIG. 5.- Universal paradigm of the disk-jet coupling for both BHXBs and AGNs. As $\dot{m}$ increases, the disk-jet system transits along the solid arrows for BHXBs and along the dashed arrows for AGNs. The tracks of transit are depicted for three representative values of $\varepsilon$, according to the result in Figure 4. Puzzling observational disk-jet features, such as why radio emission is quenched beyond a certain $\dot{m}$ and why the radio luminosity decreases (or increases) with increasing X-ray luminosity when the system is luminous (or less luminous), are explained. See the text for more details.

and relativistic jet when the source is in a steep power-law (SPL) state and being switching from the HS to the soft state (SS). We interpret that the steady outflow (first type) is associated with the disk wind and the transient relativistic jet (second type) is associated with the ergospheric jet ejected for a modest or small $\varepsilon$. Note that, as shown in the paradigm, when the entire disk become an SSD, not only that the ergospheric jet is turned off but also that the disk wind weakens, which explains the sudden drop of observed radio luminosity when the state transits to the SS. In addition, the energy of the jet per particle, $|E|$, as well as the ergospheric jet power, $L_{\text {ErgoJet }} \equiv \int \mathcal{E}^{r} d S$, increase with increasing $\dot{m}$ in a combined disk, where the surface integral $\int d S$ is carried out on a closed surface (e.g., the horizon); this likely corresponds to the observational fact that both the Lorentz factor of a jet and its radio luminosity rapidly increase with increasing $\dot{m}$ before the jet is quenched. When the BH is surrounded by a combined disk and the MHD Penrose process takes place, the relatively strong field for the inner ADAF can result in a configuration which has been studied in previous simulations (Naravan et al. 2003; Igumenshchev et al. 2003; Igumenshchev 2008; Punsly et al. 2009; McKinnev et al. 2012), here we use the name magnetcially choked accretion flow (MCAF/5 according to McKinnev et al. (2012). Accompanying by the jet formation, the formation of an MCAF can also explain some observation features when the source is in SPL. For an MCAF, because the slowed down flow can earn more time to release their heat energy via radiation, the efficiency for the flow to convert mass to radiation energy increases (Naravan et al. 2003). This can be the reason why the disk luminosity in SPL state is higher than that in HS or SS (Fender et al. 2004). In addition, the high-frequency quasi-periodic oscillation (QPO) feature observed at SPL state may be related to the jet-disk QPO mechanisms found by McKinney et al. (2012).

For AGNs, the transitions are indicated by the dashed arrows, which imply the following disk-jet couplings. First, an efficient MHD Penrose process (i.e., a greater $|E|$ ) is viable at a relatively smaller $\dot{m}$ in an AGN compared to a BHXB. Since the SSD exists only in the outer region when $\dot{m}$ is small, it is consistent with the observational fact that radio-loud AGNs favor a spectrum without a UV bump (Kawakatu et al. 2009), which possibly arises in the inner edge of an SSD. Second, the MHD Penrose process works more efficiently with increasing BH mass. This conclusion explains why AGN jets show higher Lorentz factors than BHXB jets. Moreover, it is also consistent with the observational fact (Liu et al. 2006; Chiaberge \& Marconi|2011) that most radio-loud AGN host BHs with larger masses $\left(\sim 10^{8} M_{\odot}\right)$,

5 An MCAF is formed when the accumulated magnetic field near the BH reaches the equipartition level. The strong magnetic field is able to support (or disrupt) the disk at certain radius $R_{\mathrm{m}}$, where $\varepsilon=1$. Although slowed down by the magnetospheric barrier near $R_{\mathrm{m}}$, accreting plasma can still accrete inward in the form of highly non axisymmetry, irregular streams due to magnetic Rayleigh-Taylor instability (Igumenshchev et al. 2003; Punsly et al. 2009; (McKinney et al.|2012). We stress that the magnetically dominated magnetosphere developed when the BH surrounded by a combined disk can result in an MCAF or other conceptually similar accretion flow. 
while radio-quiet AGNs host BHs with no obvious mass constraints. Third, although a BHXB jet disappears when the disk transits from a combined disk to a SSD, an AGN jet could still be launched from the ergosphere, because the $\mathrm{SSD}$ in an AGN tends to be more magnetically dominated than in a BHXB owing to a smaller mass loading, $\rho u^{r} / \bar{B}$, around a more massive $\mathrm{BH}$. We interpret that these ergospheric jets ejected from the inner edge of the thin disks in AGNs result in (at least part of) the radio-loud quasi-stellar objects; this forms a striking contrast to BHXBs from which no strong or steady radio emission has been detected in their SS. Estimated from the dynamical timescale, the characteristic time scale for an accreting $\mathrm{BH}$ system to sustain the relativistic jet, $t$, is scaled by the central $\mathrm{BH}$ mass with the relation $t \propto M_{\mathrm{BH}}$. Therefore, with $t$ equals to days for the relativistic jet from a $\mathrm{BHXB}$ with $M_{\mathrm{BH}} \sim 10 M_{\odot}$, relativistic jets from an $\mathrm{AGN}$ with $M_{\mathrm{BH}} \sim 10^{8} M_{\odot}$ may last for $\sim 10^{5}$ years.

Note also that the relation between the accretion rate, $\dot{m}$, and the ergospheric jet power, $L_{\text {ErgoJet }}$, inverses in Ranges

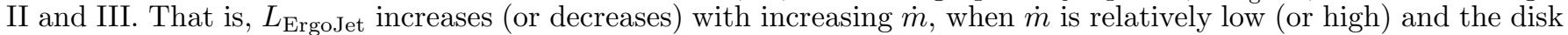
is a combined disk (or an SSD). Provided that the X-ray luminosity indicates the disk accretion rate, these conclusions naturally explain the empirical relation for both BHXBs and AGNs: the radio luminosity increases with increasing Xray luminosity when the bolometric luminosity, $L$, is small, i.e., when $L / L_{\mathrm{Edd}}=\dot{M} / \dot{M}_{\mathrm{Edd}} \sim 1 \%($ Merloni et al. 2003 ; Falcke et al. 2004), while the radio luminosity decreases with increasing X-ray luminosity when some BH systems are luminous, i.e., when $L / L_{\mathrm{Edd}} \sim 10 \%$ (King et al. 2011) .

\subsection{The Estimated Speed of Ergospheric Jet}

At large distances, the spacetime is described by the Minkowski metric; thus, the Lorentz factor of the jet, $\Gamma$, is defined by $\Gamma \equiv u^{t}$. Because $E$ is conserved quantitatively along the field lines and because the second term of Equation (13) becomes negligibly small compared to the first term at large distances, we obtain

$$
\Gamma=\frac{E_{\mathrm{out}}}{\mu},
$$

where $E_{\text {out }}$ denotes the total energy of the MHD outflow.

We can further estimate the terminal jet speed, by linking $E_{\text {out }}$ with the total energy of the MHD inflow, $E_{\text {in }}$, as:

$$
E_{\text {out }}=\frac{n_{\text {in }} u_{\text {in }}^{r}}{n_{\text {out }} u_{\text {out }}^{r}} E_{\text {in }}=-\left|\frac{n_{\text {in }} u_{\text {in }}^{r}}{n_{\text {out }} u_{\text {out }}^{r}}\right| E_{\text {in }},
$$

where the subscript "in" and "out", respectively denotes the quantities for the inflow and the outflow. The above equation is obtained by considering the conservation of the outward energy flux near the separation point, provided that the magnetic field configuration does not change significantly there. In general, the ratio of the inflow and outflow particle flux, $\left|n_{\text {in }} u_{\text {in }}^{r} / n_{\text {out }} u_{\text {out }}^{r}\right|$, is greater than unity because only a small portion of the plasmas will escape as an outflow. As a result, Equations (35) and (36) give

$$
\Gamma \geq-\frac{E_{\text {in }}}{\mu} .
$$

Hence, the inverse values of the total energy shown in Figures 4(d) and (h), as shown in Figure 6, give an estimation of the lower limit of the ergospheric jet Lorentz factor for BHXBs and AGNs. The BHXB and AGN relativistic jet speeds inferred from the observations, which has the typical value $\Gamma>2$ for BHXBs (e.g., Table 1 of Fender et al. 2004) and $\Gamma \sim 10$ for AGNs, can be consistently explained by our model.
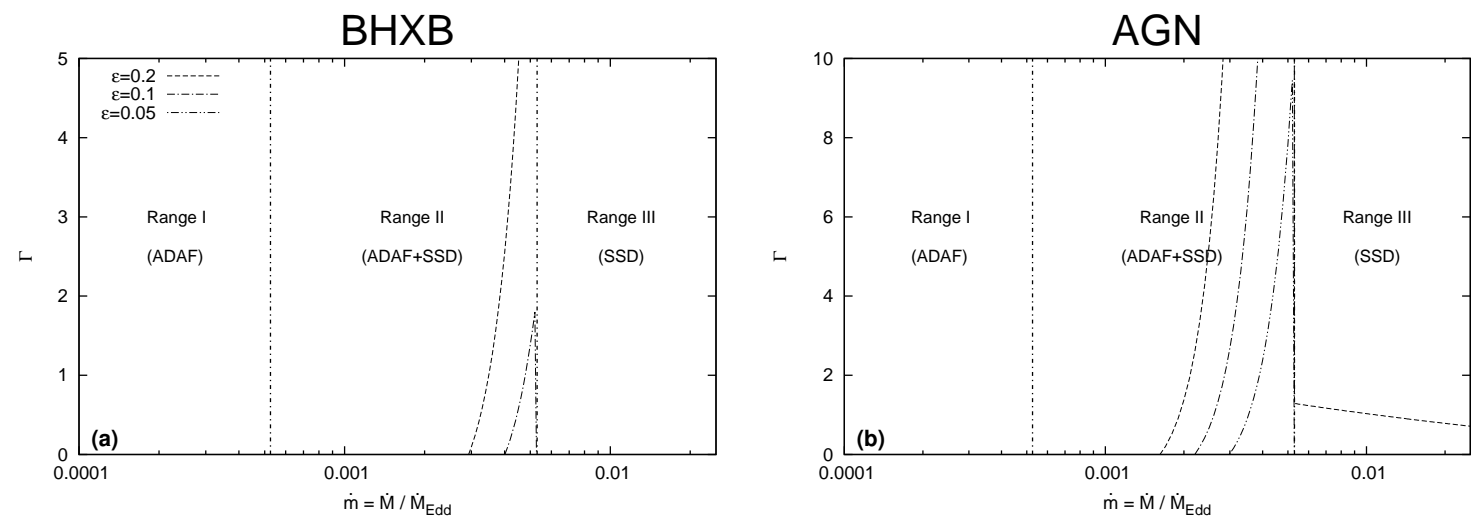

FIG. 6.- Lower limits of the Lorentz factor of ergospheric jets, $\Gamma$, modeled for BHXBs (a) and AGNs (b), as a function of the accretion rate, $\dot{m}$. See text for more details. 


\subsection{Validity of the Model}

To investigate the reason of the launching and quenching of BH relativistic jets when $\dot{m}$ varies, we solve for stationary negative energy inflow solutions (which corresponds to the formation of relativistic jets) by adopting analytical solution of the ADAF and the SSD, assuming a spherical accretion geometry near the horizon, and estimating the field strength of the large-scale hole-threading magnetic field by the parameter $\varepsilon$. The major drawback of our approach is that we ignore the possible disk instability when the SSD becomes radiation pressure dominated. In addition, our axialsymmetry and stationary solution miss the temporal and the non-axisymmetry properties of the jet formation. Also, the disk dynamo effect and the magnetic reconnection process can further modify the large-scale fields, although their effects can be in general included in the parameter $\varepsilon$.

For our purpose, we ignore several aspects which we think are minor factors in determining whether the jet is on or off for simplicity. Several discussions on the assumptions and the robustness of the result are provided in the following.

We ignore the Blandford-Znajek extraction in the force-free region near the pole of the horizon in a quasi-spherical accretion flow. However, such extraction is expected to be a relatively minor factor to determine the jet launching. The reason is that most of the hole-threading field lines have considerable plasma loading on them, and that the power for magnetic fields extracting the energy from a rotating BH is proportional to $\sin ^{2} \theta$ (Blandford \& Znajek 1977).

The most important feature of a combined disk is that the significant surface density difference of the SSD and the ADAF at the transition radius. Although here we describe the combined disk solution by patching the analytical solutions of the SSD and the ADAF at the transition radius, the above feature is expected to be true even if a more detailed disk solution is considered.

There are uncertainties in determining the large-scale magnetic fields. For example, the parameter $\varepsilon$ can vary with the radius $r$, and the total flux of the large-scale disk-threading magnetic fields is not unclear. However, the field lines that threads the disk are expected to be redistributed when the accretion disk types vary. The disk-jet coupling features described by our model can be qualitatively preserved as long as the diffuse effect is relatively unimportant than the advection effect for the large-scale disk-threading field lines. Especially, a magnetically dominated magnetosphere near the horizon is still most likely realized when the BH is surrounded by a combined disk, provided that the large-scale magnetic field advected inward from the outer SSD is large enough for the inner ADAF. Such requirement is not difficult to be satisfied as the transition radius gradually decreases to a small enough radius (Figures 4(a) and (e)).

The result presented in Figure 4 shows that a negative energy flow may form when the BH is surrounded by a combined disk (Range II). The conclusion can actually still be valid when more detailed pictures are considered. Below we discuss two possible concerns. First, we consider whether the result would change when numerical ADAF solutions, e.g., Narayan et al. (1997) and Gammie \& Popham (1998), instead of the self-similar solutions, Equations (B1)-(B3), are used. It is true that the self-similar analytical solutions are not a good approximation near the hole. For example, the causality requires a numerical solution of $u^{\mathrm{ADAF}}$ be greater than Equation (B1) near the BH, while the surface density $\sigma^{\mathrm{ADAF}}$ be less than the self-similar solution, Equation (B3). Nevertheless, the numerator in Equation (C2) will not change because of the mass conservation, $\sigma u \sim H \rho u \propto \dot{M}$, provided that the disk height $H$ remains unchanged. Since the magnetic field is predominantly supported by the outer thin disk, $\bar{B}_{\mathrm{II}}$ is essentially given by $B\left(R_{\mathrm{tr}}\right)$. As a result, neither $\mu \eta$ nor $\bar{B}_{\mathrm{II}}$ contains significant errors if we adopt the self-similar solution of ADAF. Thus, the discussion given in this paper is not vulnerable for details of the ADAF solutions. Second, we discuss whether the estimation of the mass loading is fair when $\bar{B}_{\mathrm{II}}=B\left(R_{\mathrm{tr}}\right)+B\left(R_{\mathrm{inj}}\right) \gg B\left(R_{\mathrm{inj}}\right)$. In this case, an MCAF is formed. The highly non-axisymmetry, irregular MCAF will try to avoid the strong field ranges by the magnetic Rayleigh-Taylor instability and result in strong-field, low-density 'magnetic islands' (Punsly et al. 2009). As a result, both $\rho^{\mathrm{ADAF}}$ and $u^{\mathrm{ADAF}}$ decrease in a magnetic island. Thus, even when $R_{\mathrm{m}}>R_{\mathrm{inj}}$, the mass loading from the magnetic island located at $R_{\mathrm{inj}}$ is lower than the estimate with the self-similar solutions, Equations (B1) and (B2). This means that the MHD inflow launched from the magnetic island should be even more magnetically dominated and that the energy of the inflow is still negative.

\section{CONCLUSIONS}

In this paper we focus on the fundamental question: Why relativistic jets from accreting BHs can be selectively launched under a certain accretion condition? Here we solve this problem by incorporating the plasma inertia effect, which have been neglected in previous studies, in conjunction with the electromagnetic effect on the extraction of $\mathrm{BH}$ energy. At low accretion rate (e.g., $\dot{m} \lesssim 0.1$ ), the accretion flow has a quasi-spherical geometry near the BH event horizon. As a result, whether the rotational energy of the BH can be effectively extracted outward is determined by the inward rest-mass energy of the accreting plasmas. When the outward electromagnetic energy flux dominates, a relativistic, ergospheric jet can be therefore launched; on the contrary, when the inward rest-mass energy dominates, there is no powerful relativistic jet. By examining the MHD Penrose process for BHs surrounded by different types of accretion disk at low accretion rates, we conclude that the MHD inflow becomes magnetically dominated preferentially in a combined disk, which consists of an outer SSD and an inner ADAF, to enable the launch of an ergospheric jet. Such relativistic jets are quenched if a magnetic dominance breaks down when the entire disk becomes an SSD. We also provide a universal paradigm of the coupling between the $\mathrm{BH}$ accretion disks and their relativistic jets at low accretion rates. Several observed disk-jet connections are naturally explained. Future study on the relation between the accretion geometry near the event horizon and the extraction of the $\mathrm{BH}$ energy will be important to the understanding of the $\mathrm{BH}$ jet formation mechanisms. 
We are grateful to Mike J. Cai for discussions about the relativistic Bernoulli equation. We thank C. F. Gammie, A. K. H. Kong, M. Nakamura, H. C. Spruit, R. E. Taam and anonymous referees for helpful comments and suggestions. This work was supported by the National Science Council (NSC) of Taiwan under the grant NSC 99-2112-M-007-017MY3 and the Formosa Program between NSC and Consejo Superior de Investigaciones Cientificas in Spain administered under the grant NSC100-2923-M-007-001-MY3.

\section{APPENDIX}

\section{PARAMETERIZED THE LARGE-SCALE MAGNETIC FIELD STRENGTH}

The large-scale field $B$ stored in a disk can be estimated from the point of view of energies. The large-scale field energy $E_{\mathrm{F}}$ inside the volume enclosed by a radius $R$ should not exceeds the gravitational binding energy $E_{\mathrm{G}}$ of the disk near $R$, otherwise the field would escape by buoyancy. By considering $E_{\mathrm{F}} \sim 4 \pi R^{3} B^{2} / 24$ and $\left|E_{\mathrm{G}}\right| \sim 2 \pi G M_{\mathrm{BH}} \sigma R$, we define a ratio $\varepsilon^{2}(\leq 1)$ between this two quantities to obtain

$$
B \sim \varepsilon \sqrt{12 G M_{\mathrm{BH}} \sigma / R^{2}}
$$

where $G$ is the gravitational constant, $M_{\mathrm{BH}}$ is the mass of the central $\mathrm{BH}$, and $\sigma$ is the surface density of the disk.

Alternatively, we can estimate the large-scale field $B$ stored in a disk by a force balance. If a disk accretion is supported by a large-scale field at $R$, as described in Narayan et al. (2003), the large-scale field $B$ can be estimated by the force balance $G M_{\mathrm{BH}} \sigma / R^{2} \sim B_{r} B_{\theta} / 2 \pi$ to give $B \sim \sqrt{2 \pi G M_{\mathrm{BH}} \sigma / R^{2}}$ where $B_{\theta} \sim B_{r} \sim B$ is assumed. Similarly, a parameter $\varepsilon^{2}(\leq 1)$ can be defined by the ratio between the gravity force and the field force; we thus obtain

$$
B \sim \varepsilon \sqrt{2 \pi G M_{\mathrm{BH}} \sigma / R^{2}}
$$

Since the two estimations above are consistent in order of magnitude, we simply parameterize $B$ in terms of $\varepsilon, M_{\mathrm{BH}}$, and $\sigma / R^{2}$, such that

$$
B \equiv B(\varepsilon, R, \sigma(R))=\varepsilon \sqrt{2 \pi G M_{\mathrm{BH}} \sigma / R^{2}} .
$$

ANALYTICAL SOLUTION OF THE ACCRETION DISKS

With the scaled units,

$$
\begin{gathered}
M_{\mathrm{BH}}=m \mathrm{M}_{\odot}, \\
R=\bar{r} R_{\mathrm{g}} \quad R_{\mathrm{g}} \equiv \frac{2 G M_{\mathrm{BH}}}{c^{2}}=\left(2.95 \times 10^{5} \mathrm{~m}\right) \mathrm{cm}, \\
\dot{M}=\dot{m} \dot{M}_{\mathrm{Edd}} \quad \dot{M}_{\mathrm{Edd}}=L_{\mathrm{Edd}} / 0.1 c^{2}=\left(1.39 \times 10^{18} \mathrm{~m}\right) \mathrm{g} / \mathrm{s}=\left(2.2 \times 10^{-8} \mathrm{~m}\right) \mathrm{M}_{\odot} / \mathrm{yr},
\end{gathered}
$$

we adopt the following radial velocity $u$, plasma density $\rho$, and surface density $\sigma$ solutions of an ADAF and a thin disk. Note that what we need is $\rho u$ (because $\mu \eta \equiv \rho u / B$ ) to solve the BE, and that $\rho u$ can be more or less correctly evaluated even in the innermost region by an ADAF self-similar solution without invoking a numerical solution. We therefore adopt ADAF self-similar solution (Naravan \& Yi 1995; Naravan et al. 1998) and obtain

$$
\begin{gathered}
u^{\mathrm{ADAF}}=-1.1 \times 10^{10}\left(\alpha^{\mathrm{ADAF}}\right) \bar{r}^{-\frac{1}{2}} \mathrm{cms}^{-1}, \\
\rho^{\mathrm{ADAF}}=1.31 \times 10^{-4}\left(\alpha^{\mathrm{ADAF}}\right)^{-1} m^{-1} \dot{m} \bar{r}^{-\frac{3}{2}} \mathrm{gcm}^{-3}, \\
\sigma^{\mathrm{ADAF}}=\dot{M} / 2 \pi R\left(-u^{\mathrm{ADAF}}\right)=68\left(\alpha^{\mathrm{ADAF}}\right)^{-1} \dot{m} \bar{r}^{-\frac{1}{2}} \mathrm{gcm}^{-2},
\end{gathered}
$$

while in the thin disk solutions (Shakura \& Sunyaev 1973; Kato et al. 2008), we obtain

$$
\begin{aligned}
u^{\mathrm{SSD}} & =-4.3 \times 10^{6}\left(\alpha^{S S D}\right)^{\frac{4}{5}} m^{-\frac{1}{5}} \dot{m}^{\frac{2}{5}} \bar{r}^{-\frac{2}{5}} f^{-\frac{3}{5}} \mathrm{cms}^{-1}, \\
\rho^{\mathrm{SSD}} & =20 \times\left(\alpha^{S S D}\right)^{-\frac{7}{10}} m^{-\frac{7}{10}} \dot{m}^{\frac{2}{5}} \bar{r}^{-\frac{33}{20}} f^{\frac{2}{5}} \mathrm{gcm}^{-3}, \\
\sigma^{\mathrm{SSD}} & =1.7 \times 10^{5}\left(\alpha^{S S D}\right)^{-\frac{4}{5}} m^{\frac{1}{5}} \dot{m}^{\frac{3}{5}} \bar{r}^{-\frac{3}{5}} f^{\frac{3}{5}} \quad \mathrm{gcm}^{-2},
\end{aligned}
$$

where

$$
f=\left[1-\left(\frac{\bar{r}_{\mathrm{ISCO}}}{\bar{r}}\right)^{\frac{1}{2}}\right]
$$

and $\bar{r}_{\mathrm{ISCO}}$ is the radius of the innermost stable circular orbit. Note the dimensionless coordinate, $\bar{r}$, is related to $r$ by $\bar{r}=2 r$. The parameter $\alpha$ describes the viscosity in the $\alpha$-prescription (Shakura \& Sunyaev 1973). The superscripts 
"ADAF" and "SSD" denote the Advection-dominated accretion flow and the Shakura-Sunyaev disk analytical solutions, respectively. To elucidate the general feature, we adopt typical values of the viscosity parameter such that $\alpha^{\mathrm{SSD}}=0.01$ and $\alpha^{\mathrm{ADAF}}=0.1$.

\section{SOLVING THE RELATIVISTIC BERNOULLI EQUATION FOR THE LOCATION OF THE ALFVEN POINT}

Once the field strength, field geometry, and the mass loading per field line at different $\dot{m}$ are specified, we can calculate all the coefficients in the cold $\mathrm{BE}$, Equation (26), and determine the location of the Alfven point, $R_{\mathrm{A}}$.

Since Equation (26) is solved in the geometrized unit (in length unit of $G M / c^{2}$ ), extra transformations of units from c.g.s. unit are necessary, namely,

$$
\begin{gathered}
\mathcal{H}(\text { geom. }) \simeq \bar{B}(\text { c.g.s }) \times M_{B H} G^{3 / 2} / c^{4}, \\
\mu \eta(\text { geom. }) \simeq m_{\mathrm{p}} n \frac{u}{\bar{B}}(\text { c.g.s }) \times M_{B H} G^{3 / 2} / c^{3}=\rho \frac{u}{\bar{B}}(\text { c.g.s }) \times M_{B H} G^{3 / 2} / c^{3} .
\end{gathered}
$$

These unit transformations can be done by two steps. First, we convert the unit from the c.g.s into the geometrized unit (Table 3). Second, we rescale the length unit in geometrized unit from "cm" into " $G M / c^{2}$ " (Table 4).

Next, we find the location of the Alfven point $R_{\mathrm{A}}$ at various accretion rate by the following steps:

1. Calculate the coefficients $\mathcal{A}$ and $\mathcal{B}$ in Equation (26).

2. Guess $R_{\mathrm{A}}$ to compute $E$ and $L$ by Equations (29) and (30).

3. Using $E$ and $L$, calculate the coefficients $\mathcal{C}$ and $\mathcal{D}$ in Equation (26).

4. Using the calculated $\mathcal{A}, \mathcal{B}, \mathcal{C}, \mathcal{D}$, solve the cold BE equation(26).

5 . Find $R_{\mathrm{A}}$ so that the fluid can smoothly pass through the fast-magnetosonic point.

A correct $R_{\mathrm{A}}$ will give a physical inflow solution that passes through the fast point before reaching the horizon with a nonzero speed (Figure 7). If $R_{A}$ is incorrect, the resulting inflow solution becomes unphysical, being either sub-fast-magnetosonic (Figure 8), or discontinuous (Figure 9).

TABLE 3

Unit Conversion from C.G.S. into Geometrized Unit

\begin{tabular}{cccc}
\hline \hline Quantity & c.g.s Unit & Geometrized Unit & Dimension of the Geometrized Unit \\
\hline Rest-mass energy & $\mu=m_{\mathrm{p}} c^{2}$ & $\mu$ (c.g.s $G / c^{4}=m_{\mathrm{p}}(\mathrm{c} . \mathrm{g} . \mathrm{s}) G / c^{2}$ & {$[\mathrm{~cm}]$} \\
Number density & $n$ & $n$ (c.g.s) & {$\left[\mathrm{cm}^{-3}\right]$} \\
Magnetic field & $B_{\mathrm{p}}$ & $B_{\mathrm{P}}(\mathrm{c} . \mathrm{g} . \mathrm{s}) G^{\frac{1}{2}} / c^{2}$ & {$\left[\mathrm{~cm}^{-1}\right]$} \\
Velocity & $u_{\mathrm{p}}$ & $u_{\mathrm{P}}$ (c.g.s $) / c$ & {$[1]$} \\
Mass loading & $\eta=n u_{\mathrm{p}} / B_{\mathrm{p}}$ & $\eta$ (c.g.s $c / G^{\frac{1}{2}}$ & {$\left[\mathrm{~cm}^{-2}\right]$} \\
\hline
\end{tabular}

TABLE 4

Converting the Length Unit from "cm" to " $G M / c^{2}$ "

\begin{tabular}{cccc}
\hline \hline Quantity & Geometrized Unit & Geometrized Unit Scaled by $G M / c^{2}$ & Dimension after Rescaled \\
\hline Rest-mass energy & $m_{\mathrm{p}}$ (c.g.s $G / c^{2}$ & $\left[m_{\mathrm{p}}\right.$ (c.g.s $\left.) G / c^{2}\right] /\left[\left(G M / c^{2}\right)\right]$ & {$\left[\left(G M / c^{2}\right)\right]$} \\
Number density & $n$ (c.g.s) & {$[n($ c.g.s $)] /\left[\left(G M / c^{2}\right)^{-3}\right]$} & {$\left[\left(G M / c^{2}\right)^{-3}\right]$} \\
Magnetic field & $B_{\mathrm{P}}$ (c.g.s $G^{\frac{1}{2}} / c^{2}$ & {$\left[B_{\mathrm{P}}(\right.$ c.g.s $\left.) G^{\frac{1}{2}} / c^{2}\right] /\left[\left(G M / c^{2}\right)^{-1}\right]$} & {$\left[\left(G M / c^{2}\right)^{-1}\right]$} \\
Velocity & $u_{\mathrm{P}}$ (c.g.s $/ c$ & $u_{\mathrm{P}}(\mathrm{c} . \mathrm{g} . \mathrm{s}) / c$ & {$[1]$} \\
Mass loading & $\eta$ (c.g.s $G / c^{2}$ & {$\left[\eta\right.$ c.g.s $\left.G / c^{2}\right] /\left[\left(G M / c^{2}\right)^{-2}\right]$} & {$\left[\left(G M / c^{2}\right)^{-2}\right]$} \\
\hline
\end{tabular}

\section{REFERENCES}

Abramowicz, M. A., Czerny, B., Lasota, J. P., \& Szuszkiewicz, E. 1988, ApJ, 332, 646

Abramowicz, M. A., Jaroszyński, M., Kato, S., et al. 2010, A\&A, $521, \mathrm{~A} 15$

Abramowicz, M. A., \& Zurek, W. H. 1981, ApJ, 246, 314

Blandford, R. D., \& Payne, D. G. 1982, MNRAS, 199, 883

Blandford, R. D., \& Znajek, R. L. 1977, MNRAS, 179, 433

Camenzind, M. 1986a, A\&A, 156, 137

Camenzind, M. 1986b, A\&A, 162, 32

Camenzind, M. 1987, A\&A, 184, 341

Chiaberge, M., \& Marconi, A. 2011, MNRAS, 416, 917
Esin, A. A., McClintock, J. E., \& Narayan, R. 1997, ApJ, 489, 865 Falcke, H., Körding, E., \& Markoff, S. 2004, A\&A, 414, 895

Fender, R. P., Belloni, T. M., \& Gallo, E. 2004, MNRAS, 355, 1105

Ferreira, J., Petrucci, P.-O., Henri, G., Saugé, L., \& Pelletier, G. 2006, A\&A, 447, 813

Gallo, E., Fender, R. P., \& Pooley, G. G. 2003, MNRAS, 344, 60

Gammie, C. F., \& Popham, R. 1998, ApJ, 498, 313

Hirotani, K., Takahashi, M., Nitta, S.-Y., \& Tomimatsu, A. 1992, ApJ, 386, 455

Honma, F. 1996, PASJ, 48, 77 


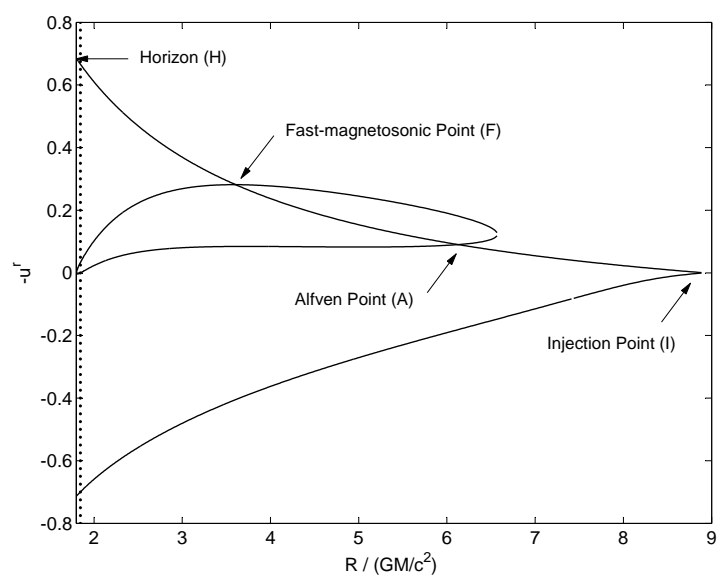

FIG. 7.- Solution of the BE, Equation (26), when $M / M_{\odot}=10, \varepsilon=0.2, \dot{m}=0.001$, and $R_{\mathrm{A}} /\left(G M / c^{2}\right)=6.128$. The BE is a fourth-order algebraic equation and it has four roots at a fixed $R$ in general. However, only the real solutions are shown here. This physical solution $(\mathrm{I} \rightarrow \mathrm{A} \rightarrow \mathrm{F} \rightarrow \mathrm{H})$ shows that the fluid is injected at the injection point (I) and passes through the Alfven (A) and the fast-magnetosonic (F) points, finally reaches the black hole $(\mathrm{H})$ with a super-fast-magnetosonic speed. The vertical dotted line indicates the location of the inner light surface. We are not interested in the outward solution $\left(-u^{r}<0\right)$, because it represents an MHD flow escaping from the BH.

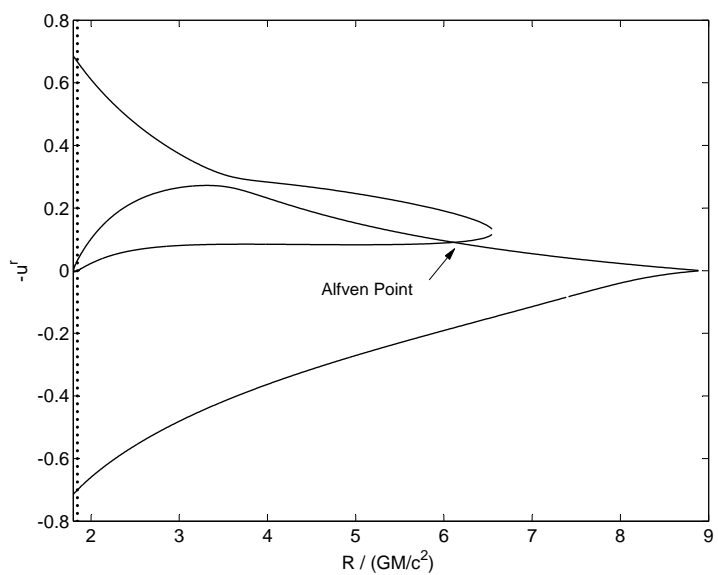

FIG. 8.- Same as Figure 7, but for $R_{\mathrm{A}} /\left(G M / c^{2}\right)=6.109$. The solution is unphysical because the trajectory starting from the injection point reaches the horizon with a vanishing inward velocity.

Igumenshchev, I. V. 2008, ApJ, 677, 317

Igumenshchev, I. V., Narayan, R., \& Abramowicz, M. A. 2003, ApJ, 592, 1042

Kato, S., Fukue, J., \& Mineshige, S. 2008, Black-Hole Accretion

Disks - Towards a New Paradigm (Kyoto: Kyoto Univ. Press)

Kawakatu, N., Nagao, T., \& Woo, J.-H. 2009, ApJ, 693, 1686

King, A. L., Miller, J. M., Cackett, E. M., et al. 2011, ApJ, 729, 19

Koide, S., Shibata, K., Kudoh, T., \& Meier, D. L. 2002, Science, 295, 1688

Komissarov, S. S. 2005, MNRAS, 359, 801

Liu, Y., Jiang, D. R., \& Gu, M. F. 2006, ApJ, 637, 669

Maccarone, T. J., Gallo, E., \& Fender, R. 2003, MNRAS, 345, L19

MacDonald, D., \& Thorne, K. S. 1982, MNRAS, 198, 345

Manmoto, T., Kato, S., Nakamura, K. E., \& Narayan, R. 2000, ApJ, 529, 127

McKinney, J. C., Tchekhovskoy, A., \& Blandford, R. D. 2012, MNRAS, 423,3083

Meier, D. L. 2005, Ap\&SS, 300, 55

Merloni, A., Heinz, S., \& di Matteo, T. 2003, MNRAS, 345, 1057

Narayan, R., Igumenshchev, I. V., \& Abramowicz, M. A. 2003, PASJ, 55, L69
Narayan, R., Kato, S., \& Honma, F. 1997, ApJ, 476, 49

Narayan, R., Mahadevan, E., Quataert, 1998, in Theory of black hole accretion discs, Accretion Around Black Holes, ed. Abramowicz, M. A., Bjornsson, G. \& Pringle, J. E.

(Cambridge: Cambridge Univ. Press), 148

Narayan, R., \& Yi, I. 1995, ApJ, 452, 710

Novikov, I. D., \& Thorne, K. S. 1973, in Black Holes, ed. C. DeWitt-Morette \& B. S. DeWitt (New York: Gordon \& Breach), 343

Penrose, R. 1969, Nuovo Cimento Riv. Ser., 1, 252

Punsly, B., Igumenshchev, I. V., \& Hirose, S. 2009, ApJ, 704, 1065

Remillard, R. A., \& McClintock, J. E. 2006, ARA\&A, 44, 49

Rothstein, D. M., \& Lovelace, R. V. E. 2008, ApJ, 677, 1221

Sadowski, A. 2009, ApJS, 183, 171

Sạdowski, A., \& Sikora, M. 2010, A\&A, 517, A18

Semenov, V., Dyadechkin, S., \& Punsly, B. 2004, Science, 305, 978

Shakura, N. I., \& Sunyaev, R. A. 1973, A\&A, 24, 337

Spruit, H. C., \& Uzdensky, D. A. 2005, ApJ, 629, 960

Takahashi, M., Nitta, S., Tatematsu, Y., \& Tomimatsu, A. 1990, ApJ, 363, 206

Trump, J. R., Impey, C. D., Kelly, B. C., et al. 2011, ApJ, 733, 60 


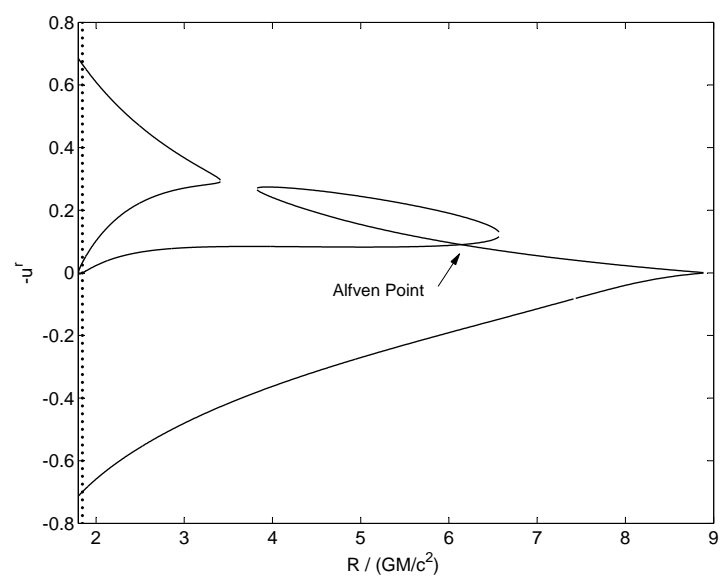

FIG. 9.- Same as Figure 7, but for $R_{\mathrm{A}} /\left(G M / c^{2}\right)=6.138$. The solution that passes the Alfven point is unphysical, because the flow cannot reach the horizon. 